\title{
Cooperative Resource Allocation Games Under Spectral Mask and Total Power Constraints
}

\author{
Jie Gao, Student Member, IEEE, Sergiy A. Vorobyov, Senior Member, IEEE, and Hai Jiang, Member, IEEE
}

\begin{abstract}
Resource allocation problems in multi-user systems, modeled as Nash bargaining (NB) cooperative games, are investigated under different constraints. Using the joint time division multiplexing and frequency division multiplexing (TDM/FDM) scheme as a manner of cooperation in the NB resource allocation game with only spectrum mask constraints (SMCs), it is shown that the efficiency of the corresponding solution depends on both the interference-to-signal and interference-to-noise ratios of the users. Sufficient conditions under which the joint TDM/FDM based NB solution outperforms a noncooperative Nash equilibrium solution are derived and shown to be mild. A semi-distributed algorithm is proposed for finding the NB solution of the corresponding game with SMCs only. The NB based resource allocation game with both SMCs and total power constraints (TPCs) is shown to be a nonconvex optimization problem of high complexity. For such a game, a classification of two-user systems into bandwidth- and power-dominant systems is proposed based on the concept of Pareto-optimality. This classification gives insights to the resource allocation problem by showing that the benefits of both users are guaranteed to increase simultaneously only when the dominant resource in the system increases. Using this classification, efficient suboptimal algorithms of low complexity are derived based on the idea of optimally allocating the dominant resource and suboptimally allocating the other resource. Simulation results demonstrate the efficiency of the proposed cooperative NB resource allocation strategies.
\end{abstract}

Index Terms-Cooperative games, multi-user systems, Nash bargaining, Pareto-optimality, spectral mask constraints, total power constraints.

\section{INTRODUCTION}

G AME theory has recently attracted a significant interest as an approach for studying resource allocation problems in the multi-user systems in which the users compete for resources and potentially conflict with each other [1]-[3]. If the users compete for resources and do not cooperate, the corresponding resource allocation games are called noncooperative games and

Manuscript received October 28, 2009; accepted April 06, 2010. Date of publication April 15, 2010; date of current version July 14, 2010. The associate editor coordinating the review of this manuscript and approving it for publication was Dr. Walid Hachem. This work was supported in part by the Natural Sciences and Engineering Research Council (NSERC) of Canada and in part by the Alberta Innovates-Technology Futures, Alberta, Canada. Part of this work were presented at the IEEE International Conference on Acoustic, Speech and Signal Processing (ICASSP), Taipei, Taiwan, April 2009, and the IEEE International Conference on Acoustic, Speech and Signal Processing (ICASSP), Dallas, TX, March 2010.

The authors are with the Department of Electrical and Computer Engineering, University of Alberta, Edmonton, AB T6G 2V4, Canada (e-mail: jgao3@ece. ualberta.ca; vorobyov@ece.ualberta.ca; hai.jiang@ece.ualberta.ca).

Color versions of one or more of the figures in this paper are available online at http://ieeexplore.ieee.org.

Digital Object Identifier 10.1109/TSP.2010.2048320 the existence and uniqueness of a so-called equilibrium of such games, typically a Nash equilibrium (NE), are usually the main concerns [4]-[8]. Noncooperative power allocation games are studied in [4]-[6] for different problem setups. The existence and uniqueness of an NE for a two-user power allocation game on a frequency selective fading channel (FSFC) are investigated in [4], while a noncooperative power allocation game on digital subscriber lines is studied in [5]. It is shown that an NE can be found in a distributed manner using iterative water-filling based algorithms. A power allocation game on multiple-input multiple-output (MIMO) multiple-access channels is studied in [6], and the existence, uniqueness, and sum-rate efficiency of an $\mathrm{NE}$ for temporal and spatial power allocations are analyzed for a two-user game. Noncooperative spectrum sharing games are considered in [7], [8]. It is shown in [7] that in the situation when multiple users coexist in the same frequency band, an NE can be nonunique, which may render the outcome of the game unpredictable. A noncooperative spectrum sharing game in a cognitive radio system consisting of a single primary and multiple secondary users is studied in [8], and it is shown that an NE is a fair yet inefficient solution for the considered problem due to the fact that the total benefit of all secondary users is not maximized. The inefficiency of an NE has also been emphasized for other games, for example, the beamforming game investigated in [9] for two multiple-antenna systems operating in the same spectrum band.

The aforementioned possible inefficiency of NE solutions has motivated the investigations of the systems with a voluntary cooperation among the users, and it has been shown that extra benefits may be obtained by the users in such systems [9]-[15]. The corresponding games are called cooperative games, and one of the most popular approaches developed for such games is the Nash bargaining (NB) approach [16]. Cooperative power allocation games are studied in [10]-[13]. A two-user power allocation game on a flat fading channel (FFC) is investigated in [10]. It is argued that certain points in the utility space of the game are not achievable from a game-theoretic perspective. The rate region achievable by the frequency division multiplexing (FDM) scheme is analyzed and the corresponding FDM based NB solution is derived. It is shown that this solution increases the benefits of all users as compared to a noncooperative NE solution. The study is extended in [11] to multi-user systems with FSFCs for the case when only spectral mask constraints (SMCs) are imposed on the users. The NB solution is derived based on the joint time division multiplexing/FDM (TDM/FDM) manner of cooperation. Unlike the FFC case, the allocation of frequency bins becomes the main concern in the FSFC case. A low-complexity algorithm is developed for finding the joint TDM/FDM based 
NB solution of the game. In [12], a nonconvex resource allocation game on an FSFC with only total power constraints (TPCs) limiting the total transmission power of each user is considered. A water-filling based algorithm is proposed to search for the NB solution in a two-user game, but the nonconvex nature of the problem leads to a high complexity of the algorithm. NB solutions are extended to games with log-convex utility spaces in [13], and it is shown that the existence and uniqueness of an NB solution in such utility spaces depend on the interference coupling among the users and, therefore, are not guaranteed in general. Other cooperative games, such as beamforming and precoding games have been considered in [9], [14], and [15].

In this paper, we consider NB based cooperative resource allocation games for multi-user systems with SMCs and TPCs. ${ }^{1}$ Our main contributions are the following. i) We show that the efficiency of the joint TDM/FDM based solutions of the considered resource allocation games with SMCs depends on both the interference-to-signal ratio (ISR) and the interference-to-noise ratio (INR) of each user. Sufficient conditions under which the joint TDM/FDM based NB solution outperforms a noncooperative NE solution are derived and shown to be mild. ii) We also show that the bargaining process among users for the NB based resource allocation games with SMCs can be physically realized in a semi-distributed manner which distributes the computations among all users. iii) We show that the NB based resource allocation game with both SMCs and TPCs corresponds to a nonconvex optimization problem which has a high complexity. We classify two-user systems into bandwidth- and power-dominant based on the concept of Pareto-optimality. Using such a classification, we develop low-complexity suboptimal algorithms for each case of bandwidth- or power-dominant systems based on the idea of optimally allocating the dominant resource and suboptimally allocating the other resource.

The rest of this paper is organized as follows. The system model and the cooperative resource allocation game formulation are given in Section II. The NB based cooperative resource allocation games with SMCs are studied in Section III. Section IV deals with the two-user NB based cooperative resource allocation games with both SMCs and TPCs. Section V demonstrates our simulation results and Section VI concludes the paper. This paper is reproducible research [19] and the software needed to generate the numerical results can be obtained from www.ece. ualberta.ca/ vorobyov/ProgNB.zip.

\section{SySTEM MODEL AND GAME FORMULATION}

\section{A. System Model}

Consider a system of $M$ users sharing $N$ frequency bins. Denote the set of user indexes as $\Omega_{M}=\{1, \ldots, M\}$ and the set of frequency bin indexes as $\Omega_{N}=\{1, \ldots, N\}$. The vector of power allocation on the frequency bins for user $i$ is written as $\mathbf{p}_{i}=\left[p_{i}(1), \ldots, p_{i}(N)\right]^{T}$ where $p_{i}(k)$ is the power that user $i$ allocates on frequency bin $k$ and $(\cdot)^{T}$ stands for the transpose.

The information rate that user $i$ can achieve is a function of all users' power allocations, and it is denoted as $r_{i}\left(\mathbf{p}_{i}, \mathbf{p}_{-i}\right)$ where $\mathbf{p}_{-i}$ stands for the combination of power allocation vectors of all

\footnotetext{
${ }^{1}$ Some preliminary results have been reported in [17] and [18].
}

users except user $i$. Treating the interference from other users as noise, the overall information rate that user $i$ can achieve is the summation of the information rates of this user on all frequency bins, that is,

$$
\begin{aligned}
& r_{i}\left(\mathbf{p}_{i}, \mathbf{p}_{-i}\right) \\
& =\sum_{k \in \Omega_{N}} \log \left(1+\frac{p_{i}(k)\left|h_{i i}(k)\right|^{2}}{\sigma_{i}^{2}(k)+\sum_{j \in \Omega_{M}, j \neq i} p_{j}(k)\left|h_{j i}(k)\right|^{2}}\right)
\end{aligned}
$$

where $h_{j i}(k)$ is the gain of the channel from transmitter $j$ to receiver $i$ on frequency bin $k$ and $\sigma_{i}^{2}(k)$ stands for the noise power for user $i$ on frequency bin $k$.

In practice, all users attempt to maximize their information rates under certain power constrains such as SMCs and optionally TPCs. SMCs are typically adopted to limit the power that users can allocate on different frequency bins. A vector of such power limits for user $i$ is $\mathbf{p}_{i}^{\max }=\left[p_{i}^{\max }(1), \ldots, p_{i}^{\max }(N)\right]^{T}$ where $p_{i}^{\max }(k)$ stands for the maximum power that user $i$ can allocate on frequency bin $k$. Although SMCs also bound the total power for user $i$ by the value $\sum_{k \in \Omega_{N}} p_{i}^{\max }(k)$, such a bound may be loose as compared to the user's total power limit $P_{i}^{\max }$. Thus, in certain cases TPCs are also needed. The SMCs and TPCs can be mathematically expressed, respectively, as

SMCs:

$$
p_{i}(k) \leq p_{i}^{\max }(k), \quad \forall i \in \Omega_{M}, \quad \forall k \in \Omega_{N}
$$

TPCs:

$$
\sum_{k \in \Omega_{N}} p_{i}(k) \leq P_{i}^{\max }, \quad \forall i \in \Omega_{M} .
$$

\section{B. Resource Allocation Game Formulation}

Considering users as players, their choices of resource allocations as strategies, and their corresponding information rates $r_{i}$ 's as utilities, the game model of the resource allocation problem can be written as

$$
\Gamma=\left\{\Omega_{M},\left\{\mathfrak{s}_{i} \mid i \in \Omega_{M}\right\},\left\{r_{i}\left(\mathfrak{s}_{i}, \mathfrak{s}_{-i}\right) \mid i \in \Omega_{M}\right\}\right\}
$$

where $\mathfrak{s}_{i}$ stands for the resource allocation strategy of user $i$ consisting of both bandwidth and power allocations ${ }^{2}$ and $\mathfrak{s}_{-i}$ stands for the combination of resource allocation strategies of all users except user $i$, and $r_{i}\left(\mathfrak{s}_{i}, \mathfrak{s}_{-i}\right)$ is the information rate that user $i$ can achieve given all users' strategies, i.e., given $\mathfrak{s}_{i}$ and $\mathfrak{s}_{-i}$.

In noncooperative games, the players (users) do not collaborate and an $\mathrm{NE}$, if it exists and it is unique, provides stable strategies for all users. An NE of a noncooperative game satisfies the following conditions

$$
r_{i}\left(\mathfrak{s}_{i}^{\mathrm{NE}}, \mathfrak{s}_{-i}^{\mathrm{NE}}\right) \geq r_{i}\left(\mathfrak{s}_{i}^{\prime}, \mathfrak{s}_{-i}^{\mathrm{NE}}\right), \quad \forall \mathfrak{s}_{i}^{\prime}, \quad \forall i \in \Omega_{M}
$$

where $\mathfrak{s}_{i}^{\mathrm{NE}}$ is the resource allocation strategy of user $i$ in the $\mathrm{NE}, \mathfrak{s}_{-i}^{\mathrm{NE}}$ is the combination of resource allocation strategies of all users except user $i$ in the NE, and $\mathfrak{s}_{i}^{\prime}$ denotes any possible resource allocation strategy of user $i$.

\footnotetext{
${ }^{2}$ The term "bandwidth allocation" is used throughout the paper to refer to the allocation of frequency bins.
} 
In cooperative games, the users are willing to cooperate with each other and agree on a common principle for sharing the public resource of the bandwidth in the system. If the users choose the NB approach as a cooperation principle, they aim at maximizing the Nash function (NF) defined in the cooperative utility space of the game as [16]

$$
\mathfrak{F}=\prod_{i \in \Omega_{M}}\left(r_{i}\left(\mathfrak{s}_{i}, \mathfrak{s}_{-i}\right)-r_{i}^{\prime}\right)
$$

where $r_{i}^{\prime}$ is the information rate (the utility) that user $i$ can achieve in a predefined disagreement point to which the users resort if the cooperation breaks up.

Although (6) defines the common principle for sharing the public resource of the bandwidth in the system, a more specific manner of cooperation according to which the bargaining is performed should be determined in cooperative NB games. It is required by the NB theory that such a particular manner of cooperation should result in a convex utility space. Note that, in our case, the utility space of the cooperative game is the $M$-user rate region ${ }^{3}$ which can be achieved based on a chosen manner of cooperation. In the literature, the users are typically assumed to cooperate with each other using orthogonal signaling schemes which allow no interference among the users. The most widely applicable orthogonal signaling approaches are the TDM, FDM, and joint TDM/FDM [3], [11], [23]. The subcarrier allocation in [3] is essentially the FDM, while [11] and [23] consider the joint TDM/FDM. We also use the joint TDM/FDM approach for the games with only SMCs in this work. There are three main reasons for considering orthogonal signaling such as the joint TDM/FDM. i) The resulted utility space of the game is convex as required by the NB theory. ii) Power-efficient simple receivers can be used since there is no interference in the system. It significantly reduces the receiver complexity, and in fact, allows for simple matched-filter receivers. As a comparison, if the users are allowed to interfere with each other in a cooperative game, they need to exchange certain information about interference to achieve a desirable performance, which may significantly increase the receiver complexity. iii) Most importantly, although the cooperative games based on orthogonal signaling (such as the joint TDM/FDM) may not achieve the boundaries of the capacity regions, the noncooperative games generally cannot achieve those boundaries either. Moreover, the solutions of the cooperative games with orthogonal signaling may outperform the solutions of the noncooperative games. It will be shown in Section III that the joint TDM/FDM based NB solutions are indeed more efficient than NE solutions under some mild conditions. It is also worth mentioning that orthogonal signaling may be inefficient when the interference is low [20]. However, it is shown in [20] that the cooperative bargaining problem becomes convex even without orthogonal signaling when the interference is low, which renders the problem simpler in this case.

For further developments, four general assumptions need to be made: i) The desired channel $h_{i i}(k)\left(\forall k \in \Omega_{N}\right)$ is

\footnotetext{
${ }^{3}$ Throughout the paper the term "rate region" refers to a region of users' rates achievable under a specified manner of cooperation, while the term "capacity region" refers to the information-theoretic capacity region which does not depend on any specific manner of cooperation.
}

known at both the transmitter and receiver sides of user $i$; ii) The TPCs are tight when they are taken into account, i.e., $P_{i}^{\max }<\sum_{k \in \Omega_{N}} p_{i}^{\max }(k)\left(\forall i \in \Omega_{M}\right)$; iii) The channel coherence time is large enough, i.e., the channel is slow fading such that the change of channel statistics is negligible within a sufficiently long period of transmission; iv) The users use codebooks approaching the Gaussian codebooks. With these assumptions, our focus is to study the cooperative resource allocation games under different sets of constraints.

\section{CoOperative Resource Allocation GAMES WITH SMCS}

\section{A. Cooperative Strategies for Two-User Games}

In this subsection, the NB based cooperative resource allocation game is considered for a two-user system, i.e., $M=2$ and $\Omega_{M=2}=\{1,2\}$. Since there is no TPC, it is easy to see that the corresponding noncooperative game has at least one NE [11]. In this $\mathrm{NE},{ }^{4}$ both users use all available frequency bins in their bandwidth allocation strategies and they use maximum allowed powers, i.e., $\mathbf{p}_{i}^{\max }=\left[p_{i}^{\max }(1), \ldots, p_{i}^{\max }(N)\right]^{T}\left(\forall i \in \Omega_{M=2}\right)$, as their power allocation strategies. Any unilateral deviation from this NE leads to the signal-to-noise ratio (SNR) degradation and, consequently, to the rate loss for the user which deviates from the NE. Note that the overall rate achieved by user $i$ in this $\mathrm{NE}$ is given as

$$
\begin{array}{r}
r_{i}^{\mathrm{NE}}=\sum_{k \in \Omega_{N}} \log \left(1+\frac{p_{i}^{\max }(k)\left|h_{i i}(k)\right|^{2}}{\sigma_{i}^{2}(k)+p_{j}^{\max }(k)\left|h_{j i}(k)\right|^{2}}\right) \\
j \neq i, \quad \forall i \in \Omega_{M=2} .
\end{array}
$$

For our cooperative game, the point $\left(r_{1}^{\mathrm{NE}}, r_{2}^{\mathrm{NE}}\right)$ in the utility space is selected as the disagreement point of the two-user game $^{5}$ and the joint TDM/FDM is chosen as the manner of cooperation (see the arguments in the previous section). This manner of cooperation suggests that any frequency bin can be used only by one user at any time instant, but it may be shared by different users throughout the operation time. The NB solution of this two-user game can be obtained by sharing at most a single frequency bin between both users [11].

The following definition is instrumental for our studies of the joint TDM/FDM based cooperative NB games.

Definition 1: The standard ordering of frequency bins in a two-user $N$-frequency bin system is such ordering that

$$
\frac{r_{2}^{\max }(m)}{r_{1}^{\max }(m)} \geq \frac{r_{2}^{\max }(n)}{r_{1}^{\max }(n)} \quad \text { if } m>n, \quad \forall m, n \in \Omega_{N}
$$

${ }^{4}$ Throughout Section III, when the term "the NE" is used, it refers to the specific NE which corresponds to the utilities (7).

${ }^{5}$ The reason we select the NE which corresponds to the utilities (7) is that it always exists for any two-user game with SMCs. Moreover, if there exists a unique NE, it is the NE which corresponds to the utilities (7). A similar selection of the disagreement point is used, for example, in [11]. Note that generally the disagreement point can be any NE if multiple NE exist or other points, for example, the origin. 
where

$$
r_{i}^{\max }(k)=\log \left(1+\frac{p_{i}^{\max }(k)\left|h_{i i}(k)\right|^{2}}{\sigma_{i}^{2}(k)}\right), \quad \forall i \in \Omega_{M=2}
$$

The result in [11] states that if the frequency bins are ordered in the standard ordering, the NB solution of the joint TDM/FDM based two-user game must satisfy the following two conditions: i) There is a frequency bin $k^{\star}$ such that $p_{1}(k)=p_{1}^{\max }(k)$, $p_{2}(k)=0, \forall k<k^{\star} ; p_{1}(k)=0, p_{2}(k)=p_{2}^{\max }(k), \forall k>k^{\star}$. This condition indicates that frequency bins 1 to $k^{\star}-1$ are allocated to user 1 exclusively while frequency bins $k^{\star}+1$ to $N$ are allocated to user 2 exclusively. ii) There is a coefficient $\alpha^{\star}$ such that the frequency bin $k^{\star}$ is allocated to user 1 for $\alpha^{\star}$ portion of time, while it is allocated to user 2 for $\left(1-\alpha^{\star}\right)$ portion of time.

In order to find $k^{\star}$ and $\alpha^{\star}$, two vectors $\alpha_{1}=$ $\left[\alpha_{1}(1), \ldots, \alpha_{1}(N)\right]^{T}$ and $\boldsymbol{\alpha}_{2}=\left[\alpha_{2}(1), \ldots, \alpha_{2}(N)\right]^{T}$ are assigned to users 1 and 2, respectively. Here $\alpha_{i}(k)$ denotes the time portion allocated to user $i$ on frequency bin $k$. Note that for the joint TDM/FDM based manner of cooperation, the bandwidth allocation strategy of user $i$ is fully represented by $\boldsymbol{\alpha}_{i}$. The power allocation strategy of user $i$, in this case, boils down to using maximum allowed power $p_{i}^{\max }(k)\left(\forall k \in \Omega_{N}\right)$ on the frequency bins allocated to the user. Then the joint TDM/FDM based NB game with SMCs can be mathematically expressed as the following optimization problem

$$
\begin{aligned}
& \max _{\left\{\boldsymbol{\alpha}_{i}\right\}} \log \left(r_{1}\left(\boldsymbol{\alpha}_{1}\right)-r_{1}^{\mathrm{NE}}\right)+\log \left(r_{2}\left(\boldsymbol{\alpha}_{2}\right)-r_{2}^{\mathrm{NE}}\right) \\
& \text { subject to : } 0 \leq \alpha_{i}(k) \leq 1, \forall i \in \Omega_{M=2}, \forall k \in \Omega_{N} \\
& \alpha_{1}(k)+\alpha_{2}(k) \leq 1, \quad \forall k \in \Omega_{N} \\
& r_{i}\left(\boldsymbol{\alpha}_{i}\right)>r_{i}^{\mathrm{NE}}, \quad \forall i \in \Omega_{M=2}
\end{aligned}
$$

where $r_{i}\left(\boldsymbol{\alpha}_{i}\right)=\sum_{k \in \Omega_{N}} \alpha_{i}(k) r_{i}^{\max }(k)$ is the rate that user $i$ can obtain given $\boldsymbol{\alpha}_{i}$ and the objective function (10) is the logarithm of the Nash function (6) for the two-user game. It is easy to see that (10)-(13) is a convex optimization problem that can be solved using standard convex optimization methods. According to the above mentioned conditions, $\alpha_{i}(k) \notin\{0,1\}$ only for $k=k^{\star}$ and $\alpha^{\star}=\alpha_{1}\left(k^{\star}\right)$ in the solution of (10)-(13).

Intuitively, the joint TDM/FDM based manner of cooperation is efficient when the interference between users is high. However, it is not trivial to quantitatively characterize the situation when the interference can be marked as high. Therefore, the following two questions are of interest: i) What are the conditions for a system to be a high-interference system? ii) Are such conditions mild enough to be satisfied in practical systems and situations?

Answering the first question, note that the joint TDM/FDM based manner of cooperation is efficient if the corresponding NB solution guarantees that the utility of each user is larger than the utility of this user in the NE solution. Consequently, the domain of the constraints in the optimization problem (10)-(13) should be nonempty. Thus, the following definition is in order.

Definition 2: A two-user system is a high-interference system if and only if the domain of the constraints in (10)-(13) is nonempty.
Therefore, if a two-user system is a high-interference system, then according to Definition 1 and the above mentioned properties of the joint TDM/FDM based NB solution, the following inequalities hold

$$
\begin{aligned}
& \sum_{k \in \Omega_{N}^{1}} \log \left(1+\frac{p_{1}^{\max }(k)\left|h_{11}(k)\right|^{2}}{\sigma_{1}^{2}(k)}\right) \\
& \quad+\alpha^{\star} \log \left(1+\frac{p_{1}^{\max }\left(k^{\star}\right)\left|h_{11}\left(k^{\star}\right)\right|^{2}}{\sigma_{1}^{2}\left(k^{\star}\right)}\right) \\
& >\sum_{k \in \Omega_{N}} r_{1}^{\mathrm{NE}}(k) \\
& \sum_{k \in \Omega_{N}^{2}} \log \left(1+\frac{p_{2}^{\max }(k)\left|h_{22}(k)\right|^{2}}{\sigma_{2}^{2}(k)}\right) \\
& \quad+\left(1-\alpha^{\star}\right) \log \left(1+\frac{p_{2}^{\max }\left(k^{\star}\right)\left|h_{22}\left(k^{\star}\right)\right|^{2}}{\sigma_{2}^{2}\left(k^{\star}\right)}\right) \\
& >\sum_{k \in \Omega_{N}} r_{2}^{\mathrm{NE}}(k)
\end{aligned}
$$

where $\Omega_{N}^{1}=\left\{1, \ldots, k^{\star}-1\right\}, \Omega_{N}^{2}=\left\{k^{\star}+1, \ldots, N\right\}$, and $r_{i}^{\mathrm{NE}}(k)$ is the rate that user $i$ can achieve on frequency bin $k$ in the NE solution, that is,

$$
r_{i}^{\mathrm{NE}}(k)=\log \left(1+\frac{p_{i}^{\max }(k)\left|h_{i i}(k)\right|^{2}}{\sigma_{i}^{2}(k)+p_{j}^{\max }(k)\left|h_{j i}(k)\right|^{2}}\right), \quad j \neq i .
$$

Inequalities (14) and (15) can be equivalently rewritten as

$$
\begin{aligned}
& \sum_{k \in \Omega_{N}^{1}} \log \left(1+\frac{p_{1}^{\max }(k)\left|h_{11}(k)\right|^{2}}{\hat{\sigma}_{1}^{2}(k)}\right) \\
& +\alpha^{\star} \log \left(1+\frac{p_{1}^{\max }\left(k^{\star}\right)\left|h_{11}\left(k^{\star}\right)\right|^{2}}{\hat{\sigma}_{1}^{2}\left(k^{\star}\right)}\right) \\
& >\sum_{k \in \Omega_{N}^{2}} r_{1}^{\mathrm{NE}}(k)+\left(1-\alpha^{\star}\right) r_{1}^{\mathrm{NE}}\left(k^{\star}\right) \\
& \sum_{k \in \Omega_{N}^{2}} \log \left(1+\frac{p_{2}^{\max }(k)\left|h_{22}(k)\right|^{2}}{\hat{\sigma}_{2}^{2}(k)}\right) \\
& \quad+\left(1-\alpha^{\star}\right) \log \left(1+\frac{p_{2}^{\max }\left(k^{\star}\right)\left|h_{22}\left(k^{\star}\right)\right|^{2}}{\hat{\sigma}_{2}^{2}\left(k^{\star}\right)}\right) \\
& >\sum_{k \in \Omega_{N}^{1}} r_{2}^{\mathrm{NE}}(k)+\alpha^{\star} r_{2}^{\mathrm{NE}}\left(k^{\star}\right)
\end{aligned}
$$

where $\hat{\sigma}_{i}^{2}(k)$ is the modified noise power for user $i$ on frequency bin $k$ defined as

$$
\begin{aligned}
\hat{\sigma}_{i}^{2}(k)= & \sigma_{i}^{2}(k)+\frac{\sigma_{i}^{4}(k)}{p_{j}^{\max }(k)\left|h_{j i}(k)\right|^{2}}+\frac{\sigma_{i}^{2}(k) p_{i}^{\max }(k)\left|h_{i i}(k)\right|^{2}}{p_{j}^{\max }(k)\left|h_{j i}(k)\right|^{2}}, \\
& j \neq i, \quad \forall k \in \Omega_{N}^{1} \cup\left\{k^{\star}\right\} \quad \text { if } i=1, \\
& \forall k \in \Omega_{N}^{2} \cup\left\{k^{\star}\right\} \quad \text { if } \quad i=2 .
\end{aligned}
$$

The right-hand sides of (17) and (18) are the "losses", for users 1 and 2, respectively, in the joint TDM/FDM based NB 
solution due to the fact that, correspondingly, user 1 can no longer use frequency bins in $\Omega_{N}^{2}$ and user 2 can no longer use frequency bins in $\Omega_{N}^{1}$. At the same time, the left-hand sides of (17) and (18) are the required "excesses" for user 1 on frequency bins in $\Omega_{N}^{1} \cup\left\{k^{\star}\right\}$ and for user 2 on frequency bins in $\Omega_{N}^{2} \cup\left\{k^{\star}\right\}$, respectively, for compensating for the "losses". Given the "losses", inequalities (17) and (18) indicate that the outcome that the NB solution is more efficient than the NE solution highly depends on the modified noise powers $\hat{\sigma}_{i}^{2}(k)$. Moreover, it follows from (19) that the level of interference for user $i$ depends on both the INRs $p_{j}^{\max }(k)\left|h_{j i}(k)\right|^{2} / \sigma_{i}^{2}(k)\left(\forall k \in \Omega_{N}\right)$ and the ISRs $p_{j}^{\max }(k)\left|h_{j i}(k)\right|^{2} / p_{i}^{\max }(k)\left|h_{i i}(k)\right|^{2}\left(\forall k \in \Omega_{N}\right)$.

Answering the second question ("Are such conditions mild enough to be satisfied in practical systems and situations?"), the following theorem is in order.

Theorem 1: If any of the following sufficient conditions

Per-user condition

$$
\begin{aligned}
& \frac{p_{j}^{\max }(k)\left|h_{j i}(k)\right|^{2}}{\sigma_{i}^{2}(k)}>\sqrt{\frac{p_{i}^{\max }(k)\left|h_{i i}(k)\right|^{2}}{\sigma_{i}^{2}(k)}+1}, \\
& \forall k \in \Omega_{N}, \quad \forall i \in \Omega_{M=2}, \quad j \neq i
\end{aligned}
$$

or Inter-user condition

$$
\begin{aligned}
& \left(1+\frac{\gamma_{k}^{0}}{1+\frac{p_{1}^{\max }(k)\left|h_{12}(k)\right|^{2}}{\sigma_{2}^{2}(k)}}\right)\left(1+\frac{\gamma_{k}^{0}}{1+\frac{p_{2}^{\max }(k)\left|h_{21}(k)\right|^{2}}{\sigma_{1}^{2}(k)}}\right) \\
& <1+\gamma_{k}^{0}, \quad \forall k \in \Omega_{N}
\end{aligned}
$$

is satisfied, then the corresponding two-user system is a highinterference system. Here

$$
\gamma_{k}^{0}=\max \left\{\frac{p_{1}^{\max }(k)\left|h_{11}(k)\right|^{2}}{\sigma_{1}^{2}(k)}, \frac{p_{2}^{\max }(k)\left|h_{22}(k)\right|^{2}}{\sigma_{2}^{2}(k)}\right\} .
$$

Proof: See Appendix A.

The following examples are of interest. Note that the conditions (20) and (21) apply on all frequency bins. Thus, only one frequency bin is considered in our examples.

Example 1: Let the SNRs for users 1 and 2 be, respectively, $p_{1}^{\max }(1)\left|h_{11}(1)\right|^{2} / \sigma_{1}^{2}(1)=31.6(\approx 15 \mathrm{~dB})$ and $p_{2}^{\max }(1)\left|h_{22}(1)\right|^{2} / \sigma_{2}^{2}(1)=10(10 \mathrm{~dB})$. According to the sufficient condition (20), if the INR for user 1 satisfies $p_{2}^{\max }(1)\left|h_{21}(1)\right|^{2} / \sigma_{1}^{2}(1)>5.7(\approx 7.6 \mathrm{~dB})$ and the INR for user 2 satisfies $p_{1}^{\max }(1)\left|h_{12}(1)\right|^{2} / \sigma_{2}^{2}(1)>3.3(\approx 5.2 \mathrm{~dB})$, then the corresponding two-user system is a high-interference system.

Example 2: Let the SNRs for users 1 and 2 be, respectively, $p_{1}^{\max }(1)\left|h_{11}(1)\right|^{2} / \sigma_{1}^{2}(1)=4(\approx 6 \mathrm{~dB})$ and $p_{2}^{\max }(1)\left|h_{22}(1)\right|^{2} / \sigma_{2}^{2}(1)=8(\approx 9 \mathrm{~dB})$. According to the sufficient condition (21), if the product of INRs satisfies $\left(p_{1}^{\max }(1)\left|h_{12}(1)\right|^{2} / \sigma_{2}^{2}(1)\right) \cdot\left(p_{2}^{\max }(1)\left|h_{21}(1)\right|^{2} / \sigma_{1}^{2}(1)\right)>\quad 9$ $(\approx 9.5 \mathrm{~dB})$, then the corresponding two-user system is a high-interference system.

It can be concluded based on Theorem 1 and the examples that conditions (20) and (21) are easy to be satisfied in practice and a majority of two-user systems are actually high-interference systems for which the joint TDM/FDM based manner of cooperation is efficient.

\section{B. Cooperative Strategies for M-User Games}

The above results can be straightforwardly generalized to $M$-user systems. Indeed, the joint TDM/FDM based $M$-user NB game with SMCs can be formulated as the following convex optimization problem

$$
\begin{gathered}
\max _{\left\{\boldsymbol{\alpha}_{i}\right\}} \sum_{i \in \Omega_{M}} \log \left(r_{i}\left(\boldsymbol{\alpha}_{i}\right)-r_{i}^{\mathrm{NE}}\right) \\
\text { subject to }: 0 \leq \alpha_{i}(k) \leq 1, \forall i \in \Omega_{M}, \forall k \in \Omega_{N} \\
\sum_{i \in \Omega_{M}} \alpha_{i}(k) \leq 1, \forall k \in \Omega_{N} \\
r_{i}\left(\boldsymbol{\alpha}_{i}\right)>r_{i}^{\mathrm{NE}}, \forall i \in \Omega_{M} .
\end{gathered}
$$

The definition of high interference extends directly from the two-user systems to the $M$-user systems. Specifically, if the domain of the constraints in (23)-(26) is nonempty, then the $M$-user system is a high-interference system. The sufficient conditions for an $M$-user system to be a high-interference system are summarized in the following theorem.

Theorem 2: If any of the following sufficient conditions

Per-user condition

$$
\sum_{j \in \Omega_{M}, j \neq i} \frac{p_{j}^{\max }(k)\left|h_{j i}(k)\right|^{2}}{\sigma_{i}^{2}(k)}>\frac{\frac{p_{i}^{\max }(k)\left|h_{i i}(k)\right|^{2}}{\sigma_{i}^{2}(k)}}{\left(1+\frac{p_{i}^{\max }(k)\left|h_{i i}(k)\right|^{2}}{\sigma_{i}^{2}(k)}\right)^{\frac{1}{M}}-1}-1,
$$

or Inter-user condition

$$
\prod_{i \in \Omega_{M}}\left(1+\frac{\gamma_{k}^{0}}{1+\frac{\sum_{j \in \Omega_{M}, j \neq i} p_{j}^{\max }(k)\left|h_{j i}(k)\right|^{2}}{\sigma_{i}^{2}(k)}}\right)<1+\gamma_{k}^{0},
$$

is satisfied, then the corresponding $M$-user system is a highinterference system. Here

$$
\gamma_{k}^{0}=\max \left\{\frac{p_{1}^{\max }(k)\left|h_{11}(k)\right|^{2}}{\sigma_{1}^{2}(k)}, \ldots, \frac{p_{M}^{\max }(k)\left|h_{M M}(k)\right|^{2}}{\sigma_{M}^{2}(k)}\right\} .
$$

The proof of this theorem is a direct extension of the proof of Theorem 1 and it is omitted here.

The following two examples are of interest. Here again only one frequency bin is considered.

Example 3: Consider a four-user system with the users' SNRs $p_{1}^{\max }(1)\left|h_{11}(1)\right|^{2} / \sigma_{1}^{2}(1)=8$ $(\approx 9 \mathrm{~dB}), \quad p_{2}^{\max }(1)\left|h_{22}(1)\right|^{2} / \sigma_{2}^{2}(1)=16 \quad(\approx 12$ $\mathrm{dB}), \quad p_{3}^{\max }(1)\left|h_{33}(1)\right|^{2} / \sigma_{3}^{2}(1)=4 \quad(\approx 6 \mathrm{~dB})$, and $p_{4}^{\max }(1)\left|h_{44}(1)\right|^{2} / \sigma_{4}^{2}(1)=10(10 \mathrm{~dB})$. According to the sufficient condition (27), if the users' INRs satisfy $\sum_{j \neq 1} p_{j}^{\max }(1)\left|h_{j 1}(1)\right|^{2} / \sigma_{1}^{2}(1)>9.9(\approx 10 \mathrm{~dB})$, $\sum_{j \neq 2} p_{j}^{\max }(1)\left|h_{j 2}(1)\right|^{2} / \sigma_{2}^{2}(1)>14.5(\approx 11.6 \mathrm{~dB})$, $\sum_{j \neq 3} p_{j}^{\max }(1)\left|h_{j 3}(1)\right|^{2} / \sigma_{3}^{2}(1)>7.1(\approx 8.5 \mathrm{~dB})$, and 
$\sum_{j \neq 4} p_{j}^{\max }(1)\left|h_{j 4}(1)\right|^{2} / \sigma_{4}^{2}(1)>11.2(\approx 10.5 \mathrm{~dB})$, then the system is a high-interference system.

Example 4: Consider a system of six users with the maximum SNR $31.6(\approx 15 \mathrm{~dB})$. According to the inter-user condition, the system is a high-interference system if the left-hand side of $(28)$ is smaller than $32.6(\approx 15 \mathrm{~dB})$. Particularly, assume that the INRs $\sum_{j \neq i} p_{j}^{\max }(1)\left|h_{j i}(1)\right|^{2} / \sigma_{i}^{2}(1)\left(\forall i \in \Omega_{M}\right)$ are the same for all users for the sake of evaluation, then the required INR is $\sum_{j \neq i} p_{j}^{\max }(1)\left|h_{j i}(1)\right|^{2} / \sigma_{i}^{2}(1)>39.3(\approx 15.9 \mathrm{~dB})$ for each user. The average required INR from each interfering user is $7.9(\approx 9.0 \mathrm{~dB})$.

From the above examples, it can be seen that the requirements of the conditions (27) and (28) on the average INR from each interferer are not difficult to be satisfied. The above examples verify again that the conditions for an $M$-user system to be a high-interference system are mild. Thus, the joint TDM/FDM based manner of cooperation can be considered as a valid approach which is generally efficient.

Unlike the two-user case, there is no fast algorithm as in [10] available in the literature for finding the NB solution for the $M$-user case. Therefore, it is necessary to design an algorithm realizing the bargaining process in the $M$-user game. Note that we do not assume the availability of an additional independent central point for the considered $M$-user system. Indeed, if there is a central point, which can do the computations in order to find the optimal cooperative solution for all $M$ users, there is no need for bargaining among the users. Therefore, bargaining is especially desirable when there is no central point available in the system. Moreover, none of the users has the computational resource or the wish to perform the function of such a central point. Thus, we next propose a semi-distributed algorithm for solving (23)-(26), which distributes the major computations among all users. Note that a coordinator is still required in the proposed semi-distributed algorithm. However, the complexity of the problem that must be solved by a coordinator is much lower than the complexity of the original problem (23)-(26), and therefore, any user has a sufficient computational resource to serve as a coordinator.

\section{Semi-Distributed Algorithm for Finding the NB Solution}

The problem (23)-(26) is a convex optimization problem with coupling constraints $\sum_{i \in \Omega_{M}} \alpha_{i}(k) \leq 1\left(\forall k \in \Omega_{N}\right)$. Therefore, it can be solved in a semi-distributed manner using the dual decomposition method [21].

The Lagrange dual problem to the problem (23)-(26) is given as

$$
\begin{gathered}
\max _{\left\{\boldsymbol{\alpha}_{i}\right\}} \sum_{i \in \Omega_{M}} \log \left(r_{i}\left(\boldsymbol{\alpha}_{i}\right)-r_{i}^{\mathrm{NE}}\right) \\
-\sum_{k \in \Omega_{N}} \lambda(k)\left(\sum_{i \in \Omega_{M}} \alpha_{i}(k)-1\right) \\
\text { subject to: } \quad 0 \leq \alpha_{i}(k) \leq 1, \forall i \in \Omega_{M}, \forall k \in \Omega_{N} \\
r_{i}\left(\boldsymbol{\alpha}_{i}\right)>r_{i}^{\mathrm{NE}}, \forall i \in \Omega_{M} \\
\lambda(k) \geq 0, \forall k \in \Omega_{N}
\end{gathered}
$$

where $\lambda(k)$ is a nonnegative Lagrange multiplier on frequency bin $k$. All Lagrange multipliers form the $N \times 1$ vector $\lambda=$ $[\lambda(1), \ldots, \lambda(N)]^{T}$.

The problem (30)-(33) can be further converted into a twolevel optimization problem with the following lower level subproblems:

$$
\begin{gathered}
\max _{\boldsymbol{\alpha}_{i}} \log \left(r_{i}\left(\boldsymbol{\alpha}_{i}\right)-r_{i}^{\mathrm{NE}}\right)-\sum_{k \in \Omega_{N}} \lambda(k) \alpha_{i}(k) \\
\text { subject to : } \quad 0 \leq \alpha_{i}(k) \leq 1, \quad \forall k \in \Omega_{N} \\
r_{i}\left(\boldsymbol{\alpha}_{i}\right)>r_{i}^{\mathrm{NE}}
\end{gathered}
$$

for each user $i \in \Omega_{M}$, and the higher level master problem

$$
\begin{aligned}
& \min _{\lambda} \sum_{i \in \Omega_{M}} U_{i}(\boldsymbol{\lambda})+\sum_{k \in \Omega_{N}} \lambda(k) \\
& \text { subject to: } \quad \lambda(k) \geq 0, \quad \forall k \in \Omega_{N}
\end{aligned}
$$

where $U_{i}(\lambda)$ is the maximum value of the objective function (34) given $\lambda$.

The two-level dual problem (34)-(36) and (37)-(38) can be solved based on a semi-distributed structure with a coordinator. Since the original problem is convex, strong duality holds and the solutions of the dual problem (30)-(33) and the original problem (23)-(26) are the same if Slater's condition is satisfied [22]. For our specific problem, we have the following result.

Theorem 3: The Slater's condition is guaranteed to be satisfied for the problem (23)-(26) as long as the NB solution exists.

Proof: See Appendix A.

Substituting $r_{i}\left(\boldsymbol{\alpha}_{i}\right)=\sum_{k \in \Omega_{N}} \alpha_{i}(k) r_{i}^{\max }(k)$ into the objective function of the subproblem (34)-(36), this subproblem can be rewritten as

$$
\begin{array}{cc}
\max _{\boldsymbol{\alpha}_{i}} & \log \left(\sum_{k \in \Omega_{N}} \alpha_{i}(k) r_{i}^{\max }(k)-r_{i}^{\mathrm{NE}}\right) \\
- & \sum_{k \in \Omega_{N}} \lambda(k) \alpha_{i}(k) \\
\text { subject to }: \quad 0 \leq \alpha_{i}(k) \leq 1, \quad \forall k \in \Omega_{N} \\
\sum_{k \in \Omega_{N}} \alpha_{i}(k) r_{i}^{\max }(k)>r_{i}^{\mathrm{NE}} .
\end{array}
$$

The lower level subproblems (39)-(41) can be solved distributively by the corresponding users. Moreover, information required for solving the $i$ th subproblem, that is, $r_{i}^{\max }(k)$ 's $(\forall k \in$ $\left.\Omega_{N}\right)$ and $r_{i}^{\mathrm{NE}}$, is local to user $i$.

The algorithm for solving the dual problem is summarized in Table I. The complexity of finding the NB solution is determined by the complexity of the lower level subproblems (39)-(41), which is $O\left(N^{3}\right)$. Note that the coefficients $\lambda(k)$ 's $\left(k \in \Omega_{N}\right)$ have specific physical meanings. Indeed, $\lambda(k)$ represents the risk that cooperation among users breaks up due to a conflict on sharing frequency bin $k$. Thus, in the lower level subproblems, the objective for each user consists of two parts. Generally, a larger $\alpha_{i}(k)$ is preferred to increase the total information rate of user $i$. However, if $\alpha_{i}(k)$ becomes too large, the cooperation may break up and the utility of user $i$ will return to the inferior noncooperative solution. 
TABLE I

DuAl DECOMPOSITION ALGORITHM FOR THE JOINT TDM/FDM BASED NB GAME WITH SMCS

1. The coordinator initializes $\lambda=\lambda^{0}=$ $\left[\lambda^{0}(1), \lambda^{0}(2), \ldots, \lambda^{0}(N)\right]^{T}$ and broadcasts it to all users.

2. Each user solves (39)-(41) according to the present $\lambda$ and transmits its solutions for $\alpha_{i}(k)$ 's $\left(\forall k \in \Omega_{N}\right)$ to the coordinator.

3. The coordinator updates $\boldsymbol{\lambda}$ according to the gradient of the master problem (37)-(38) as $\hat{\lambda}(k)=[\lambda(k)-\delta(1-$ $\left.\left.\sum_{i \in \Omega_{M}} \alpha_{i}(k)\right)\right]_{+}\left(\forall k \in \Omega_{N}\right)$ where $(\cdot)_{+}$denotes the projection onto non-negative sub-space and $\delta$ is the step length of the algorithm.

4. If $|\hat{\lambda}(k)-\lambda(k)| \leq \xi\left(\forall k \in \Omega_{N}\right)$, stop; otherwise the coordinator broadcasts $\hat{\lambda}=[\hat{\lambda}(1), \hat{\lambda}(2), \ldots, \hat{\lambda}(N)]^{T}$ and go to step 2 . Here, $\xi$ is the stopping threshold of the algorithm.

\section{Cooperative Resource Allocation Games With SMCS AND TPCS FOR TWO USERS}

The presence of TPCs significantly changes the resource allocation problem. Indeed, under TPCs, the users may not be able to use maximum powers on their allocated frequency bins if the joint TDM/FDM based manner of cooperation is considered. Therefore, both $\alpha_{i}(k)$ 's and $p_{i}(k)$ 's $\left(i \in \Omega_{M}, k \in \Omega_{N}\right)$, representing the users' bandwidth allocation strategies and power allocation strategies, respectively, have to be optimized jointly in this case. Thus, with both SMCs and TPCs, the joint TDM/FDM based NB problem can be formulated as the following optimization problem

$$
\max _{\left\{\boldsymbol{\alpha}_{i}\right\},\left\{\mathbf{p}_{i}\right\}} \sum_{i \in \Omega_{M}} \log \left(r_{i}\left(\boldsymbol{\alpha}_{i}, \mathbf{p}_{i}\right)-r_{i}^{\prime}\right)
$$

subject to : $0 \leq \alpha_{i}(k) \leq 1, \forall i \in \Omega_{M}, \forall k \in \Omega_{N}$

$$
\begin{aligned}
& \sum_{i \in \Omega_{M}} \alpha_{i}(k) \leq 1, \forall k \in \Omega_{N} \\
& r_{i}\left(\boldsymbol{\alpha}_{i}, \mathbf{p}_{i}\right)>r_{i}^{\prime}, \forall i \in \Omega_{M} \\
& \sum_{k \in \Omega_{N}} \alpha_{i}(k) p_{i}(k) \leq P_{i}^{\max }, \forall i \in \Omega_{M} \\
& p_{i}(k) \leq p_{i}^{\max }(k), \forall i \in \Omega_{M}, \forall k \in \Omega_{N}
\end{aligned}
$$

where $r_{i}\left(\boldsymbol{\alpha}_{i}, \mathbf{p}_{i}\right)$ is the overall information rate that user $i$ can obtain under the power allocation strategy $\mathbf{p}_{i}$ and bandwidth allocation strategy $\boldsymbol{\alpha}_{i}$ given as

$$
\begin{aligned}
r_{i}\left(\boldsymbol{\alpha}_{i}, \mathbf{p}_{i}\right) & =\sum_{k \in \Omega_{N}} r_{i}(k) \\
& =\sum_{k \in \Omega_{N}} \alpha_{i}(k) \log \left(1+\frac{p_{i}(k)\left|h_{i i}(k)\right|^{2}}{\sigma_{i}^{2}(k)}\right) .
\end{aligned}
$$

The optimization problem (42)-(47) is a nonconvex problem. Indeed, the Hessian matrix $\mathbf{H}_{f_{i}}$ of $f_{i}\left(\boldsymbol{\alpha}_{i}, \mathbf{p}_{i}\right)=$ $\sum_{k \in \Omega_{N}} \alpha_{i}(k) p_{i}(k)$ can be written as

$$
\mathbf{H}_{f_{i}}=\nabla^{2} f_{i}\left(\boldsymbol{\alpha}_{i}, \mathbf{p}_{i}\right)=\left(\begin{array}{cc}
\mathbf{0} & \mathbf{I} \\
\mathbf{I} & \mathbf{0}
\end{array}\right)
$$

where $\mathbf{I}$ and $\mathbf{0}$ denote the identity and all-zero matrices, respectively. Thus, $\mathbf{H}_{f_{i}}$ 's $\left(\forall i \in \Omega_{M}\right)$ are orthogonal matrices, i.e., $\mathbf{H}_{f_{i}} \mathbf{H}_{f_{i}}^{T}=\mathbf{I}\left(\forall i \in \Omega_{M}\right)$. The eigenvalues of an orthogonal matrix can only be 1 or -1 . Moreover, it is known that the summation of all eigenvalues of $\mathbf{H}_{f_{i}}$ equals the trace of $\mathbf{H}_{f_{i}}$ which is zero for any $i$. Therefore, any $\mathbf{H}_{f_{i}}\left(\forall i \in \Omega_{M}\right)$ must have equal number of eigenvalues 1 and -1 , which means that $\mathbf{H}_{f_{i}}$ 's $\left(\forall i \in \Omega_{M}\right)$ are indefinite. Thus, the constraints $\sum_{k \in \Omega_{N}} \alpha_{i}(k) p_{i}(k) \leq P_{i}^{\max }\left(\forall i \in \Omega_{M}\right)$ are nonconvex and the nonconvexity of the optimization problem (42)-(47) follows.

In the following studies, the two-user case is considered. The disagreement point is chosen at the origin of the utility space, that is, $r_{i}^{\prime}=0\left(\forall i \in \Omega_{M=2}\right)$, instead of the NE point as was in the case of the resource allocation problem with only SMCs. The disagreement point is chosen at the origin because finding a noncooperative NE solution in the game with both SMCs and TPCs is itself a complicated problem.

\section{A. Bandwidth-Dominant and Power-Dominant Systems}

Finding the joint TDM/FDM based NB solution of the problem (42)-(47) requires joint power and bandwidth allocations for each user, and the resulted complexity of the two-user game can be unacceptably high. Moreover, the joint TDM/FDM based manner of cooperation with maximum power allocation as adopted for games with only SMCs can be inefficient in some cases when TPCs are present. To overcome these problems, we categorize systems into two classes and deal with each class of system differently. Toward this end, two definitions need to be given first.

Definition 3: A point $\mathbf{x}$ is Pareto-optimal in space $\mathcal{S}$ if and only if $\mathbf{y}=\mathbf{x}$ for all $\mathbf{y}$ satisfying $\mathbf{y} \succeq \mathbf{x}$ in $\mathcal{S}$, where $\succeq$ denotes the element-wise inequality.

A Pareto-optimal point corresponds to an efficient allocation of system resources. The NB solution is one of the Pareto-optimal points in a utility space.

For the two-user cooperative game, there exists an algorithm in the literature for obtaining the joint TDM/FDM based NB solution if SMCs are the only constraints [10] (see also [11] and [23]). The idea of this algorithm is of interest for our further studies. For any given integer $\hat{k} \in \Omega_{N}$, let

$$
\begin{aligned}
& \alpha_{1}(k)=1, \quad \alpha_{2}(k)=0, \quad p_{1}(k)=p_{1}^{\max }(k), \\
& p_{2}(k)=0, \quad \forall k<\hat{k} \\
& \alpha_{1}(k)=0, \quad \alpha_{2}(k)=1, \quad p_{1}(k)=0, \\
& p_{2}(k)=p_{2}^{\max }(k), \quad \forall k>\hat{k} \\
& \alpha_{1}(\hat{k})=\beta, \quad \alpha_{2}(\hat{k})=1-\beta, \quad p_{1}(\hat{k})=p_{1}^{\max }(\hat{k}), \\
& p_{2}(\hat{k})=p_{2}^{\max }(\hat{k}) .
\end{aligned}
$$

If the frequency bins are in the standard ordering defined in (8), then the corresponding point $\left(r_{1}\left(\boldsymbol{\alpha}_{1}\right), r_{2}\left(\boldsymbol{\alpha}_{2}\right)\right)$ is guaranteed to be Pareto-optimal in the joint TDM/FDM based rate region for any $0 \leq \beta \leq 1$. Varying $\hat{k}$ and $\beta$, all Pareto-optimal points can be obtained including the NB solution of the game. In other words, the NB solution for the two-user cooperative resource allocation game with only SMCs can be found by searching on the Pareto-boundary instead of the entire utility space of the game, where the Pareto-boundary is defined as the following.

Definition 4: All Pareto-optimal points in a convex space $\mathcal{S}$ form the Pareto-boundary of $\mathcal{S}$.

The above mentioned algorithm in [10] is based on the principle that frequency bins which are "better" for a certain user 
should be allocated to this user prior to frequency bins which are "inferior". However, this principle may fail and lead to highly inefficient solutions if TPCs are also imposed. Consider the following simple example.

Example 5: Assume that there are four frequency bins and $\left[r_{1}^{\max }(k), r_{2}^{\max }(k)\right]^{T}$ are $[0.5,0.1]^{T}$ for $k=1,[2,1]^{T}$ for $k=2,[1,3]^{T}$ for $k=3$, and $[0.3,1]^{T}$ for $k=4$. Also assume that $\mathbf{p}_{i}^{\max }=[1,1,1,1]^{T}$ and $P_{i}^{\max }=1.5$ for either user. Then according to the aforementioned principle [10], the following resource allocation can be obtained: $\boldsymbol{\alpha}_{1}=[1,0.5,0,0]^{T}, \boldsymbol{\alpha}_{2}=[0,0,0.5,1]^{T}$, $\mathbf{p}_{1}=[1,1,0,0]^{T}$ and $\mathbf{p}_{2}=[0,0,1,1]^{T}$. Note that the TPCs $\sum_{k \in \Omega_{N}} \alpha_{i}(k) p_{i}(k) \leq P_{i}^{\max }\left(i \in \Omega_{M=2}\right)$ are used to derive the coefficients $\alpha_{1}(2)=\left(P_{1}^{\max }-p_{1}^{\max }(1) \alpha_{1}(1)\right) / p_{1}^{\max }(2)=0.5$ and $\alpha_{2}(3)=\left(P_{2}^{\max }-p_{2}^{\max }(4) \alpha_{2}(4)\right) / p_{2}^{\max }(3)=0.5$. The resulted rates are $r_{1}=1.5$ and $r_{2}=2.5$, and obviously the point $(1.5,2.5)$ is not Pareto-optimal. For example, the strategies $\boldsymbol{\alpha}_{1}=[0.5,1,0,0]^{T}, \boldsymbol{\alpha}_{2}=[0,0,1,0.5]^{T}, \mathbf{p}_{1}=[1,1,0,0]^{T}$, and $\mathbf{p}_{2}=[0,0,1,1]^{T}$ provide higher rates for both users.

It follows from the above discussion that the presence of TPCs renders a different bargaining problem since there is a need to coordinate the power allocation and the bandwidth allocation. Therefore, a different approach has to be developed. Toward this end, we first consider the solutions for the NB game with only SMCs (denoted as game $\mathcal{G} 1$ ), and then add TPCs to the game (denoted as game $\mathcal{G} 2$ ).

Observation 1: TPCs do not enlarge the utility space of the game. The Pareto-optimal solutions for game $\mathcal{G} 1$ are also Pareto-optimal for game $\mathcal{G} 2$ if they are achievable.

Denote the Pareto-boundary of the joint TDM/FDM utility space of game $\mathcal{G} 1$ as $\mathcal{P}_{1}$. Then, the following result is in order.

Theorem 4: Assume that the frequency bins are in the standard ordering defined in (8). A nonempty subset of $\mathcal{P}_{1}$ can be achieved in game $\mathcal{G} 2$ under both SMCs and TPCs if and only if there exist $1 \leq \tilde{k} \leq N$ and $0 \leq \tilde{\alpha} \leq 1$ such that

$$
\frac{P_{1}^{\max }-\sum_{k=1}^{\tilde{k}-1} p_{1}^{\max }(k)}{p_{1}^{\max }(\tilde{k})} \geq \tilde{\alpha} \geq \frac{\sum_{k=\tilde{k}}^{N} p_{2}^{\max }(k)-P_{2}^{\max }}{p_{2}^{\max }(\tilde{k})}
$$

Proof: See Appendix B.

According to (53), all two-user systems can be categorized into bandwidth- and power-dominant systems. If condition (53) is satisfied, the system is bandwidth-dominant and the rates of both users are guaranteed to increase simultaneously only when new frequency bins are added into the system. Otherwise, the system is power-dominant and the rates of both users are guaranteed to increase simultaneously only when TPCs of the users are relaxed.

Observation 2: Beginning as bandwidth-dominant, a twouser system gradually changes towards power-dominant as the number of available frequency bins increases.

\section{B. Bandwidth-Dominant Systems: Joint TDM/FDM Based Bargaining}

In the bandwidth-dominant systems, the joint TDM/FDM based manner of cooperation is efficient in the sense that a nonempty subset of $\mathcal{P}_{1}$ can be achieved in game $\mathcal{G} 2$. Denote the Pareto-boundary of the joint TDM/FDM utility space of game $\mathcal{G} 2$ as $\mathcal{P}_{2}$. Then, for the bandwidth-dominant systems, the bargaining can be restricted in the set $\mathcal{P}^{\prime}=\mathcal{P}_{2} \cap \mathcal{P}_{1}$ only. The resulted NB solution, denoted as $S_{N B}^{\prime}$, can be suboptimal as compared to the optimal solution of the nonconvex optimization problem (42)-(47). It is because the power allocation (which is not the dominant factor in this case) is not optimized jointly with bandwidth allocation.

Denote the optimal NB solution of game $\mathcal{G} 2$ as $S_{N B}^{o p t}$. Also denote the joint TDM/FDM utility spaces of games $\mathcal{G} 1$ and $\mathcal{G} 2$ as $\mathcal{U}_{1}$ and $\mathcal{U}_{2}$, respectively. Then, the following theorem about the optimality of $S_{N B}^{\prime}$ is in order.

Theorem 5: $S_{N B}^{\prime}=S_{N B}^{o p t}$ if $S_{N B}^{o p t} \in \mathcal{P}^{\prime}$. If $S_{N B}^{\prime} \neq S_{N B}^{o p t}$, then $S_{N B}^{\text {opt }} \notin \mathcal{P}_{1}$ but $S_{N B}^{\prime} \in \mathcal{P}_{1}$, which means that $S_{N B}^{o p t}$ is not Pareto-optimal in $\mathcal{U}_{1}$ but $S_{N B}^{\prime}$ is Pareto-optimal in $\mathcal{U}_{1}$.

Proof: See Appendix B.

Theorem 5 leads to the following two conclusions about the optimality of $S_{N B}^{\prime}$ in the bandwidth-dominant systems: (i) $S_{N B}^{\prime}$ can be identical to the optimal joint TDM/FDM based NB solution; (ii) $S_{N B}^{\prime}$ is guaranteed to be Pareto-optimal in $\mathcal{U}_{1}$ (which is larger than $\mathcal{U}_{2}$ ) even if the optimal NB solution is not Paretooptimal in $\mathcal{U}_{1}$. Theorem 5 also indicates that for bandwidthdominant systems, the same algorithm as the one developed for finding the NB solution of the two-user game without TPCs can be used, with $\mathcal{P}_{1}$ replaced by $\mathcal{P}^{\prime}$. Therefore, an efficient solution, in the sense of Pareto-optimality, can be obtained with a low complexity.

Observe that the Pareto-boundary $\mathcal{P}_{1}$ of $\mathcal{U}_{1}$ is a piece-wise linear boundary. The slop of the $k$ th line segment $l_{k}$ of $\mathcal{P}_{1}$ is $-r_{2}^{\max }(k) / r_{1}^{\max }(k)\left(k \in \Omega_{N}\right)$ where $r_{1}$ is the horizontal axis and $r_{2}$ is the vertical axis. There are $N-1$ junctions $J_{1}, \ldots, J_{N-1}$ on $\mathcal{P}_{1}$. Let the rates associated with junction $J_{t}$ be denoted as $r_{1}^{J_{t}}$ and $r_{2}^{J_{t}}$ for users 1 and 2, respectively. Then the following theorem gives a sufficient condition under which the result $S_{N B}^{\prime}=S_{N B}^{o p t}$ holds.

Theorem 6: For bandwidth-dominant systems with standard ordering of frequency bins defined in (8), a sufficient condition for $S_{N B}^{\prime}=S_{N B}^{o p t}$ is that for given total power limits $P_{1}^{\max }$ and $P_{2}^{\max }$, the inequality $r_{1}^{d} \leq r_{1}^{\star} \leq r_{1}^{u}$ is satisfied. Here $r_{1}^{\star}$ is the abscissa of the point $\left(r_{1}^{\star}, r_{2}^{\star}\right)$ in the rate region satisfying

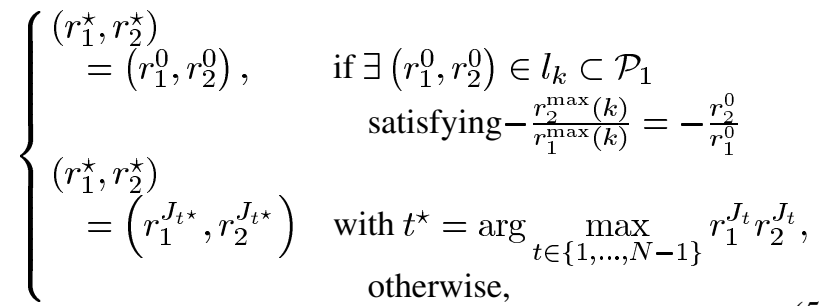

and

$$
\begin{aligned}
r_{1}^{d}=\sum_{k=1}^{\breve{k}-2} r_{1}^{\max }(k)+\left(1-\frac{1}{p_{2}^{\max }(\breve{k}-1)}\right. \\
\left.\quad \times\left(P_{2}^{\max }-\sum_{k=\breve{k}}^{N} p_{2}^{\max }(k)\right)\right) r_{1}^{\max }(\breve{k}-1)
\end{aligned}
$$


with $\breve{k}$ being the minimum integer in the interval $[1, N]$ satisfying

$$
P_{2}^{\max }-\sum_{k=\breve{k}}^{N} p_{2}^{\max }(k) \geq 0
$$

while

$$
r_{1}^{u}=\sum_{k=1}^{\bar{k}} r_{1}^{\max }(k)+\frac{1}{p_{1}^{\max }(\bar{k}+1)}\left(\begin{array}{c}
P_{1}^{\max }-\sum_{k=1}^{\bar{k}} p_{1}^{\max }(k) \\
\times r_{1}^{\max }(\bar{k}+1)
\end{array}\right.
$$

with $\bar{k}$ being the maximum integer in the interval $[1, N]$ satisfying

$$
P_{1}^{\max }-\sum_{k=1}^{\bar{k}} p_{1}^{\max }(k) \geq 0 .
$$

Proof: See Appendix B.

The following two examples further illustrate Theorem 6 .

Example 6: Consider a system with $N=3$ frequency bins in standard ordering with $r_{1}^{\max }(1)=1, r_{1}^{\max }(2)=1, r_{1}^{\max }(3)=$ $5 / 9, r_{2}^{\max }(1)=1 / 3, r_{2}^{\max }(2)=1$, and $r_{2}^{\max }(3)=5 / 3$. A Nash curve, that is, $r_{1} \cdot r_{2}=121 / 36$, is tangent to the rate region at the point $\left(r_{1}, r_{2}\right)=(11 / 6,11 / 6)$ on the line segment $l_{2}$ (see also the proof of Theorem 6 in Appendix B for details). Then according to Theorem 6, a sufficient condition for $S_{N B}^{\prime}=S_{N B}^{o p t}$ is that $P_{1}^{\max } \geq p_{1}^{\max }(1)+5 p_{1}^{\max }(2) / 6$ and $P_{2}^{\max } \geq p_{2}^{\max }(3)+$ $p_{2}^{\max }(2) / 6$.

Example 7: Consider a system with $N=3$ frequency bins in standard ordering with $r_{1}^{\max }(1)=1 / 3, r_{1}^{\max }(2)=1 / 3$, $r_{1}^{\max }(3)=4 / 9, r_{2}^{\max }(1)=1 / 6, r_{2}^{\max }(2)=1 / 3$, and $r_{2}^{\max }(3)=4 / 3$. A Nash curve, that is, $r_{1} \cdot r_{2}=8 / 9$, intersects the rate region at the unique point $\left(r_{1}, r_{2}\right)=(2 / 3,4 / 3)$, which is the junction connecting the line segments $l_{2}$ and $l_{3}$ (see also the proof of Theorem 6 in Appendix B for details). Then according to Theorem 6, a sufficient condition for $S_{N B}^{\prime}=S_{N B}^{o p t}$ is that $P_{1}^{\max } \geq p_{1}^{\max }(1)+p_{1}^{\max }(2)$ and $P_{2}^{\max } \geq p_{2}^{\max }(3)$.

\section{Power-Dominant Systems: the Sampled Joint FDM/Time Sharing Based Bargaining}

Let us now consider the case of power-dominant systems. Example 5 in Section IV-A is, in fact, an example of a power-dominant system. From this example, we can make the following observation.

Observation 3: In power-dominant systems, the use of the maximum allowed power on all allocated frequency bins generally results in a nonoptimal solution for game $\mathcal{G} 2$.

To verify this observation, let us denote the set of all frequency bins as $\mathcal{B}$, the subset of frequency bins which user 1 occupies using the maximum allowed power as $\mathcal{B}_{1}^{\max }$, the subset of frequency bins which user 2 occupies using the maximum allowed power as $\mathcal{B}_{2}^{\max }$. Then, user 1 can improve its rate by water-filling on the frequency bins $\mathcal{B}-\mathcal{B}_{2}^{\max }$ instead of using the maximum allowed power on $\mathcal{B}_{1}^{\max }$, while the rate of user 2 can be kept the same. Here $\mathcal{B}-\mathcal{B}_{2}^{\max }$ denotes the difference between sets $\mathcal{B}$ and $\mathcal{B}_{2}^{\max }$, and the general term water-filling is used to represent the specific meaning of finding the solution of the following convex optimization problem

$$
\begin{aligned}
\max _{\mathbf{p}_{i}} & \sum_{k \in \Omega_{N}} \log \left(1+\boldsymbol{\varepsilon}_{i}(k) p_{i}(k)\right) \\
\text { subject to }: & \sum_{k \in \Omega_{N}} p_{i}(k)=P_{i}^{\max } \\
& p_{i}(k) \leq p_{i}^{\max }(k), \quad \forall k \in \Omega_{N}
\end{aligned}
$$

which is a single-user multi-frequency bin power allocation problem with constant $\varepsilon_{i}(k)=\left|h_{i i}(k)\right|^{2} / \sigma_{i}^{2}(k)$ being a measure of channel $k$ 's quality for user $i$.

Observation 3 suggests that the power-dominant games have to be played based on a different manner of cooperation from the joint TDM/FDM with maximum allowed powers on all allocated frequency bins. The crux here is that the power allocation, which is the dominant factor for power-dominant systems, has the priority. A reasonable choice of the manner of cooperation for the power-dominant systems is then the joint FDM/time sharing (TS). The main idea of the joint FDM/TS is to use the TS between points corresponding to different FDM based bandwidth allocations, while performing the power allocation using the water-filling (59)-(61). Note that the power allocation based on water-filling is optimal for any given bandwidth allocation. Unfortunately, the complexity of finding the joint FDM/TS based NB solution, in this case, is very high, especially when the number of frequency bins is large. To obtain the joint FDM/TS based NB solution, the water-filling should first be performed for all $2^{N}$ possible bandwidth allocations, and the resulted $2^{N}$ points in the utility space should be recorded. Then the TS is used to obtain a minimum convex space containing all these points and the NB solution is derived within this convex space. The complexity of the TS is $O\left(4^{N}\right)$, which is exponential in the number of frequency bins.

To reduce the complexity, we consider a sampled version of the joint FDM/TS, which is the sampled joint FDM and TS (SJ-FDM/TS). According to the SJ-FDM/TS approach, a suboptimal solution of the game can be found using the algorithm summarized in Table II. In the first step of the algorithm, two sets of frequency bins which are most desirable for users 1 and 2 are found and denoted as $\tilde{\mathcal{B}}_{1}$ and $\tilde{\mathcal{B}}_{2}$, respectively. Here, the set of frequency bins $\tilde{\mathcal{B}}_{i}$ is found by performing the water-filling for user $i$ on the entire set of frequency bins $\mathcal{B}$. Then, the set of frequency bins which are desired by both users is $\mathcal{B}_{c}=\tilde{\mathcal{B}}_{1} \cap \tilde{\mathcal{B}_{2}}$, and the allocation of the frequency bins from $\mathcal{B}_{c}$ is the key of the bandwidth allocation.

In the second step, the frequency bins from $\mathcal{B}_{c}$ are allocated in a manner which favors user 1 and the corresponding FDM samples are obtained. Specifically, all frequency bins from $\mathcal{B}_{c}$ are allocated to user 1 in the first round. Then, a frequency bin with smallest channel gain for user 1 is transferred to user 2 at each subsequent round of the iterative procedure which consists of $L=\left|\mathcal{B}_{c}\right|$ rounds where $\left|\mathcal{B}_{c}\right|$ denotes the cardinality of the set $\mathcal{B}_{c}$. Let us denote the set of frequency bins which have been transferred from user 1 to user 2 during rounds 1 to $j$ as $\mathcal{B}_{s}^{j}$ where $j$ stands for the current round. In round $j$, user 1 performs water-filling on the set $\mathcal{B}-\mathcal{B}_{s}^{j}$ and selects the frequency 
TABLE II

THE SJ-FDM/TS BASED ALGORITHM FOR THE POWER-DOMINANT SysteMS

$\overline{\overline{1 .} \text { Both users perform water-filling (59)-(61) on } \mathcal{B} \text { and obtain the sets }}$ $\tilde{\mathcal{B}}_{i}\left(i \in \Omega_{M=2}\right)$. Then, $\mathcal{B}_{c}=\tilde{\mathcal{B}_{1}} \cap \tilde{\mathcal{B}}_{2}$ is found.

2. In the first round of this step, user 1 is allocated the set of frequency bins $\tilde{\mathcal{B}}_{1}$ and user 2 performs water-filling on $\mathcal{B}-\tilde{\mathcal{B}}_{1}$. In round $j(j \in$ $\{1, \ldots, L\})$, user 1 selects a subset $\mathcal{B}_{s}^{j}$ of $j-1$ frequency bins with smallest channel gains from $\mathcal{B}_{c}$ and performs water-filling on $\mathcal{B}-\mathcal{B}_{s}^{j}$. Then, user 2 performs water-filling on the remaining frequency bins. After $L$ rounds, $L$ points in the utility space are obtained.

3. Similar to Step 2, user 2 is initially allocated the set of frequency bins $\tilde{\mathcal{B}_{2}}$ and user 1 performs water-filling on $\mathcal{B}-\tilde{\mathcal{B}}_{2}$. In round $j(j \in$ $\{1, \ldots, L\})$, user 2 selects a subset $\mathcal{B}_{s}^{j}$ of $j-1$ frequency bins with smallest channel gains from $\mathcal{B}_{c}$ and performs water-filling on $\mathcal{B}-\mathcal{B}_{s}^{j}$, while user 1 performs water-filling on the remaining frequency bins. After $L$ rounds, other $L$ points in the utility space are obtained.

4. The set of $2 L$ points obtained in Steps 2 and 3 is denoted as $\mathcal{T}$ and the Pareto-boundary $\mathcal{P}_{\mathcal{T}}$ of $\mathcal{S}_{\mathcal{T}}$, which is the minimum convex space containing $\mathcal{T}$, is obtained.

5. The bargaining on $\mathcal{P}_{\mathcal{T}}$ is performed and the solution $S_{N B}^{\prime \prime}$ is obtained.

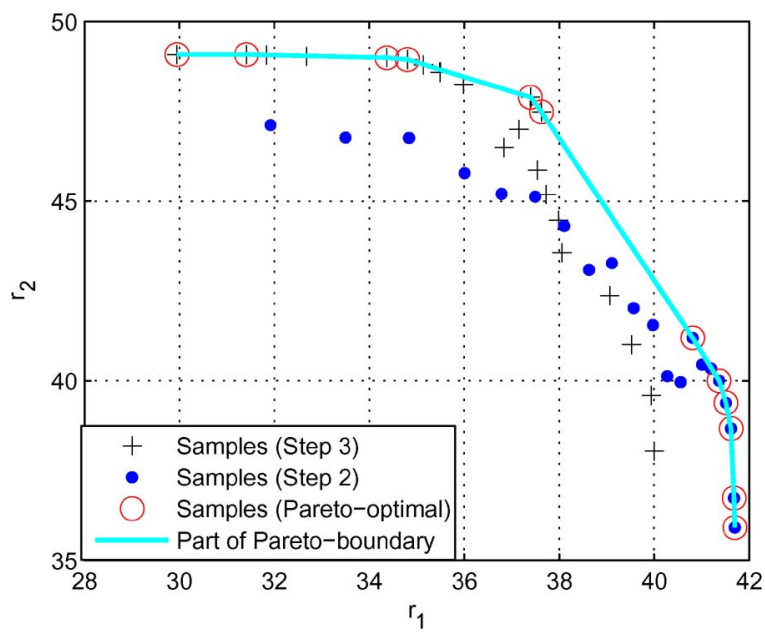

Fig. 1. The graphical demonstration of the principle used in the proposed SJ-FDM/TS based algorithm for power-dominant systems.

bins (from $\mathcal{B}-\mathcal{B}_{s}^{j}$ ) to which it allocates nonzero power according to the corresponding water-filling solution. Then user 2 performs water-filling on the frequency bins which are not claimed by user 1 and selects the frequency bins to which it allocates nonzero power. The above bandwidth allocation favors user 1 in two ways. First, user 1 always obtains the frequency bins from $\mathcal{B}_{c}$ with largest channel gains. Second, user 1 always has a priority to obtain any other frequency bins which are not in $\mathcal{B}_{c}$. In this step, we obtain $L$ points in the utility space $\mathcal{U}_{2}$ (see Fig. 1, the 'dots'). Each of these points corresponds to the highest rate that user 1 can obtain given that it can have only $L-j+1$ frequency bins from $\mathcal{B}_{c}$ where $j \in\{1, \ldots, L\}$ is the round index.

The third step of the algorithm is similar to the second step with the only difference that the frequency bins are allocated in a manner which favors user 2 . As a result, we obtain $L$ more points in the utility space $\mathcal{U}_{2}$ (see Fig. 1 , the ' + ' symbols). Note that while obtaining these $2 L$ points in Steps 2 and 3, the power allocation is strictly based on water-filling.

In the fourth step, the minimum convex utility space $\mathcal{S}_{\mathcal{T}}$, which contains the set $\mathcal{T}$ of all $2 L$ points obtained in Steps 2
TABLE III

THE OVERALl ALgORITHM FOR THE Two-User NB GAME WITH SMCs AND TPCs

\begin{tabular}{l}
\hline 1. Check the condition (53): If it is satisfied, go to step 2, \\
otherwise, go to step 3 . \\
$\overline{\text { 2. System is bandwidth-dominant: Search on the Pareto-boundary }}$ \\
$\mathcal{P}^{\prime}$, and return the solution $S_{N B}^{\prime}$. \\
\hline 3. System is power-dominant: Derive $\tilde{\mathcal{B}}_{1}, \tilde{\mathcal{B}}_{2}$, and $\mathcal{B}_{c}$. Play the \\
$2 L$ rounds and obtain $\mathcal{T}$ and $\mathcal{P}_{\mathcal{T}}$. Search on $\mathcal{P}_{\mathcal{T}}$, and return the \\
solution $S_{N B}^{\prime \prime}$.
\end{tabular}

and 3 , is found. The Pareto-boundary of the utility space $\mathcal{S}_{\mathcal{T}}$ is $\mathcal{P}_{\mathcal{T}}$ (see Fig. 1, the circled points and the curve). Finally, in the last step, the NB is performed on $\mathcal{P}_{\mathcal{T}}$ and the solution of the game is obtained.

This algorithm gives the priority to the power allocation as the water-filling, which is optimal for any given bandwidth allocation, is used. The bandwidth allocation, which is not the dominant factor for the power-dominant systems, is performed according to the principle described in Steps 2 and 3, which is motivated by the necessity of reducing the complexity. Thus, focusing on the dominant power resource, the algorithm can provide an efficient solution with low complexity. It is worth noting that the algorithm is more efficient when $L$ is relatively small as compared to $N$. Moreover, the algorithm actually finds the optimal solution when $L=0$. The efficiency of the algorithm is studied next.

Let $\mathcal{W F}^{i}(\mathcal{X})$ denotes the water-filling operator for user $i$ on the set of frequency bins $\mathcal{X}$. It returns the maximum rate that user $i$ can obtain by optimizing its power allocation on $\mathcal{X}$. Let also the point $\mathbf{r}^{\text {opt }}$ corresponding to the optimal FDM/TS based NB solution $S_{N B}^{o p t}$ be obtained by TS of two points $\left(r_{1}^{o p t 1}, r_{2}^{o p t 1}\right)$ and $\left(r_{1}^{\text {opt } 2}, r_{2}^{\text {opt }}\right)$ in the utility space $\mathcal{U}_{2}$ of game $\mathcal{G} 2$ with TS coefficients $\lambda$ and $1-\lambda$, respectively, that is, $\mathbf{r}^{o p t}=\left(\lambda r_{1}^{o p t 1}+\right.$ $\left.(1-\lambda) r_{1}^{o p t 2}, \lambda r_{2}^{o p t 1}+(1-\lambda) r_{2}^{o p t 2}\right)$. Denote the sets of frequency bins allocated to the users in the points $\left(r_{1}^{o p t 1}, r_{2}^{o p t 1}\right)$ and $\left(r_{1}^{\text {opt } 2}, r_{2}^{\text {opt } 2}\right)$ as $\left(\mathcal{B}_{1}^{\text {opt } 1}, \mathcal{B}_{2}^{\text {opt } 1}\right)$ and $\left(\mathcal{B}_{1}^{\text {opt } 2}, \mathcal{B}_{2}^{\text {opt } 2}\right)$, respectively. Then, the following theorem is in order.

Theorem 7: The SJ-FDM/TS based NB solution $S_{N B}^{\prime \prime}$ obtained using the algorithm in Table II can be identical to the FDM/TS based NB solution $S_{N B}^{o p t}$. If they are not identical, the difference $d$ between the logarithm of the NF for $S_{N B}^{o p t}$ and the logarithm of the NF for $S_{N B}^{\prime \prime}$ is bounded by

$$
d<\min \left(\log \left(\frac{\mathcal{W F}^{2}\left(\mathcal{B}_{2}^{\text {opt } 2}\right)}{\mathcal{W F}^{2}\left(\mathcal{B}-\tilde{\mathcal{B}}_{1}\right)}\right), \log \left(\frac{\mathcal{W F}^{1}\left(\mathcal{B}_{1}^{\text {opt } 1}\right)}{\mathcal{W F}^{1}\left(\mathcal{B}-\tilde{\mathcal{B}}_{2}\right)}\right)\right) .
$$

Proof: See Appendix B.

The following conclusions can be drawn: (i) $S_{N B}^{\prime \prime}$ can be identical to $S_{N B}^{o p t}$; (ii) The efficiency of $S_{N B}^{\prime \prime}$ depends on the $\operatorname{ratios} \mathcal{W F}^{i}\left(\mathcal{B}_{i}^{o p t i}\right) / \mathcal{W} \mathcal{F}^{i}\left(\mathcal{B}-\tilde{\mathcal{B}}_{j}\right)\left(j \neq i, \forall i \in \Omega_{M=2}\right)$.

\section{The Two-User Algorithm}

The overall algorithm, which combines both the bandwidthdominant and power-dominant cases, for the two-user cooperative NB game with both SMCs and TPCs is given in Table III.

In the bandwidth-dominant case, the complexity of searching on $\mathcal{P}^{\prime}$ is $O(N)$. In the power-dominant case, the complexity of 
the algorithm in Table II is determined by the TS part, which is $O\left(L^{2}\right)$. In the latter case, the complexity reduction as compared to $O\left(4^{N}\right)$ for the optimal FDM/TS based solution (where the time consumed on water-filling is neglected in both cases) is significant, especially for large $N$.

\section{Simulation Results}

\section{A. Cooperative Resource Allocation Games With SMCs}

In the first example, we assume that two users share four available frequency bins. The noise power $\sigma_{i}^{2}(k)$ is 0.01 for both users on all frequency bins. The channel gains of the desired channels $h_{11}(k)\left(\forall k \in \Omega_{N}\right)$ and $h_{22}(k)\left(\forall k \in \Omega_{N}\right)$ are generated as Rayleigh random variables with mean 1 . The channel gains of the interfering channels $h_{12}(k)\left(\forall k \in \Omega_{N}\right)$ and $h_{21}(k)\left(\forall k \in \Omega_{N}\right)$ are generated as Rayleigh random variables with means 0.7 and 0.2 , respectively. The elements of the spectral mask vector $\mathbf{p}_{i}^{\max }\left(\forall i \in \Omega_{M}\right)$ are also random variables with mean 1 .

In Fig. 2(a), the NB solution is shown together with the NE solution. The boundary of the joint TDM/FDM based rate region is also included in the figure. Fig. 2(b) displays the values of the logarithm of the NF under different TDM/FDM based bandwidth allocations. In this subfigure, $k$ is the frequency bin being shared and $\alpha$ is the fraction of time that user 1 uses frequency bin $k$. It can be seen in Fig. 2(a) that the NB solution lies on the boundary of the joint TDM/FDM based rate region and provides significantly larger rates to both users than the NE solution. Moreover, the NB solution is fair to both users. In Fig. 2(b), the largest value of the logarithm of the NF corresponds to the optimal scheme that provides the NB solution.

In the second example, the semi-distributed algorithm for the $M$-user game developed in Section III-C is tested. It is assumed that four users share six frequency bins. The channel gains of the desired and interfering channels are generated as Rayleigh random variables with means 1 and 0.2 , respectively. The elements of the spectral mask vector $\mathbf{p}_{i}^{\max }\left(\forall i \in \Omega_{M}\right)$ are also random variables with mean 1 . The step length is $\delta=0.2$ (if not otherwise specified) and the stopping threshold is $\xi=10^{-5}$.

The iterations of the NB process are shown in Fig. 3(a). The four curves on the upper side of Fig. 3(a) show the instantaneous information rates that the corresponding users can achieve, and the curve at the bottom shows the corresponding values of the logarithm of the NF. The NB and NE solutions and the comparison between them in terms of the percentage of improvement provided by the NB solution versus the NE solution are shown in Table IV for one of the runs. It can be seen from Fig. 3(a) and Table IV that all users obtain supplementary benefit from cooperation. The corresponding final allocation of time portions on each frequency bin for each user is shown in Fig. 3(b). It can be seen that frequency bins $1,2,3$, and 4 are occupied exclusively by users $3,4,1$, and 2, respectively, while frequency bins 5 and 6 are shared by users 1 and 4, and users 2 and 3, respectively.

Fig. 3(c) depicts the effect of the step length on the convergence speed of the algorithm. With the step lengths $\delta \in$ $\{0.1,0.2,0.3\}$, the corresponding logarithm of the NF is shown. It can be seen that the algorithm is time-efficient with a good choice of the step length.

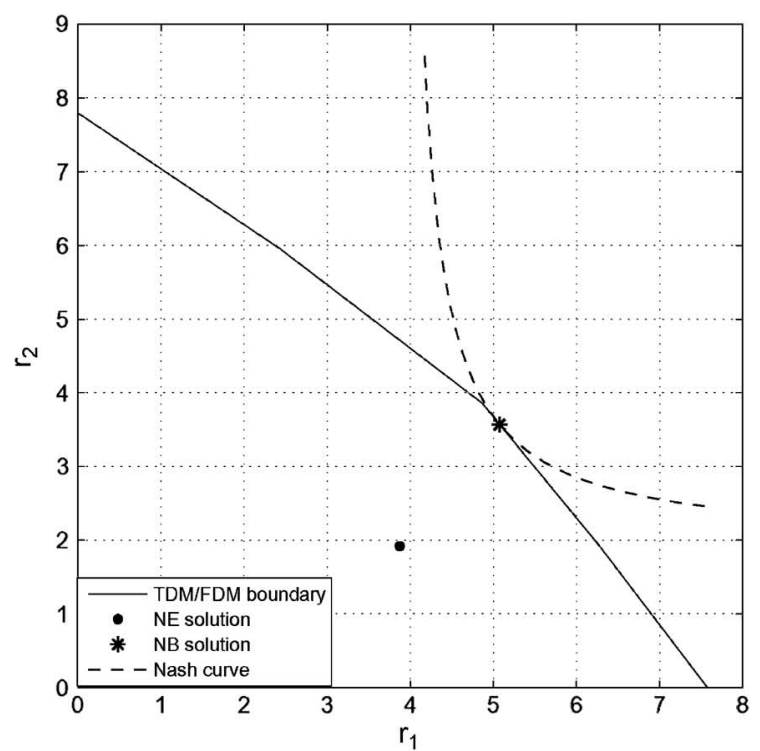

(a)

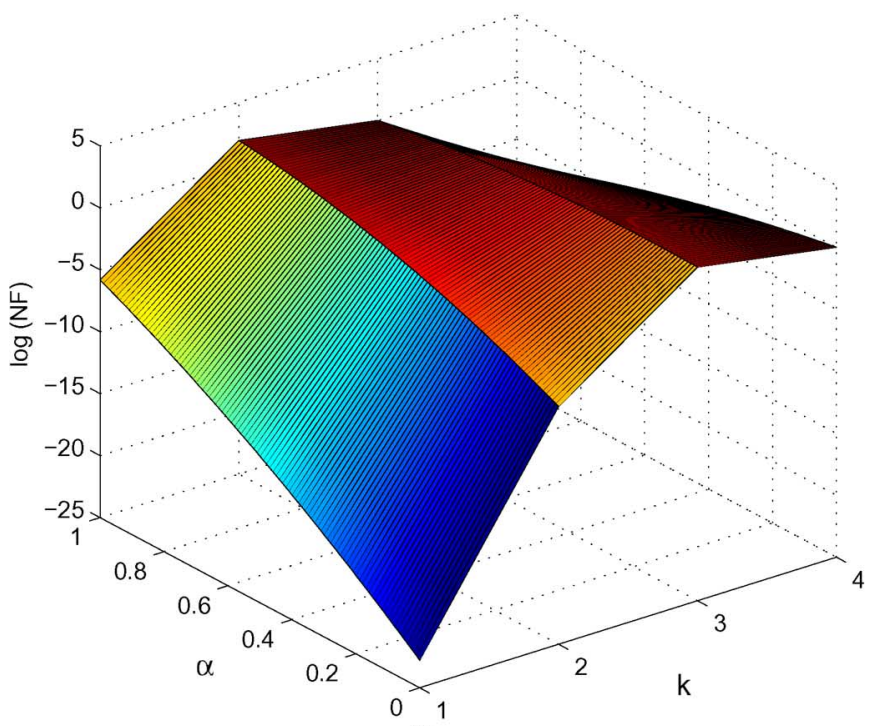

(b)

Fig. 2. Numerical example 1: (a) the TDM/FDM based rate region and the NE and NB solutions; (b) $\log (\mathrm{NF})$ under different TDM/FDM based bandwidth allocations.

\section{B. Cooperative Resource Allocation Games With SMCs and TPCs}

Fig. 4 shows the system classification according to Theorem 4 versus the total power limits and the number of frequency bins for a two-user system. The total power limits of the users $P_{1}^{\max }$ and $P_{2}^{\max }$ vary in the interval $P^{\max } \in[1,51]$. The number of frequency bins $N$ increases from 1 to 256. The desired channel gains are randomly generated using Rayleigh distribution with mean 1, and the users do not interfere with each other due to the orthogonal signaling assumption. The power limits on different frequency bins $p_{i}^{\max }(k)\left(\forall i \in \Omega_{M}, \forall k \in \Omega_{N}\right)$ are uniformly distributed in the interval $[1.8,2.2]$. The frequency bins are in the standard ordering (see (8)). Following the principle introduced in [10] (see Section IV-A), the maximum number of frequency bins $k_{i}$ that user $i$ can cover is the maximum integer in $\Omega_{N}$ that satisfies $\sum_{k=1}^{k_{1}} p_{1}^{\max }(k) \leq P^{\max }$ for $k_{1}$ and $\sum_{k=N-k_{2}+1}^{N} p_{2}^{\max }(k) \leq P^{\max }$ for $k_{2}$. The total normalized 

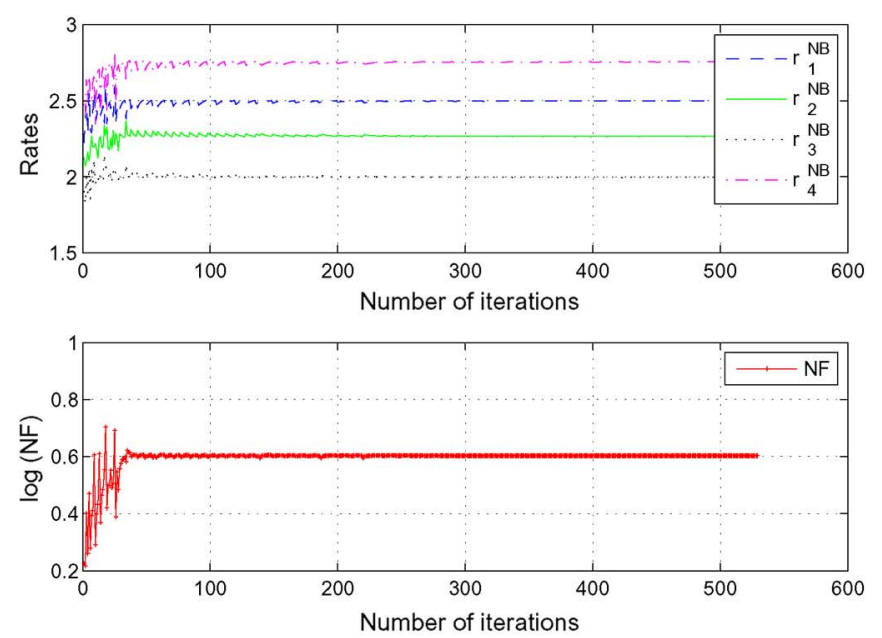

(a)

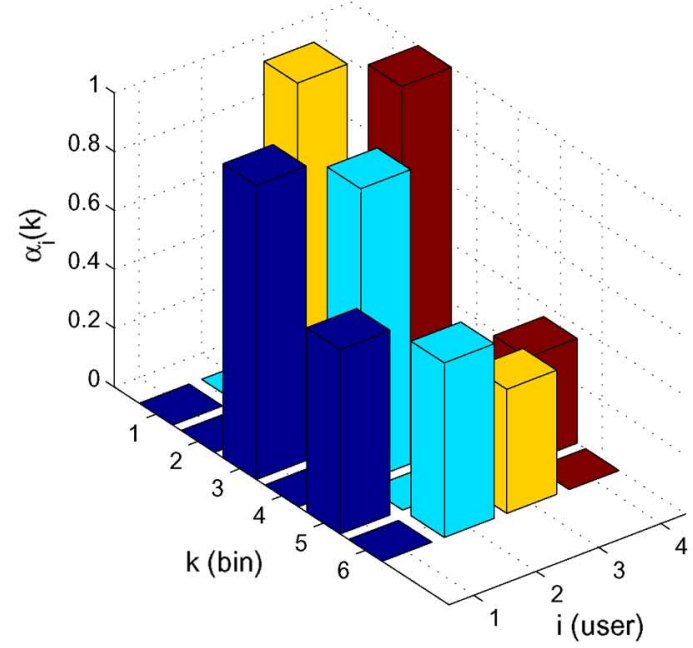

(b)
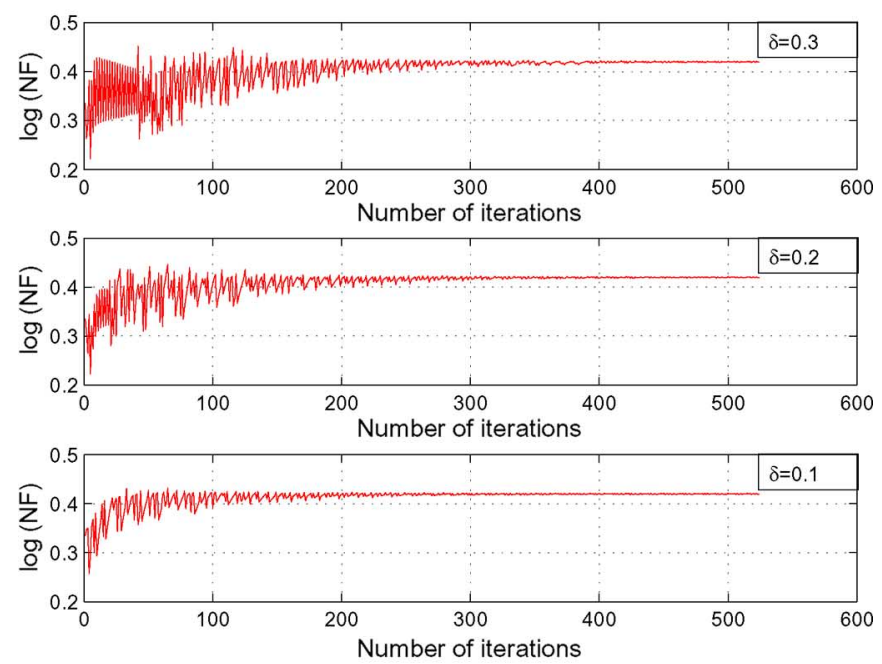

(c)

Fig. 3. Numerical example 2: (a) instantaneous information rates and the corresponding logarithm of the NF versus the number of iterations; (b) frequency bin allocations $\left\{\alpha_{i}(k)\right\}$; (c) $\log (\mathrm{NF})$ versus the number of iterations for $\delta \in$ $\{0.3,0.2,0.1\}$.

bandwidth $b_{i}$ (with the bandwidth of each frequency bin normalized to 1$)$ that user $i$ can cover is then $b_{1}=k_{1}+\left(P^{\max }-\right.$ $\left.\sum_{k=1}^{k_{1}} p_{1}^{\max }(k)\right) / p_{1}^{\max }\left(k_{1}+1\right)$ for user 1 and $b_{2}=k_{2}+\left(P^{\max }-\right.$
TABLE IV

COMPARISONS BETWEEN NE AND NB

\begin{tabular}{r||c|c|c}
\hline User & NE Solution & NB solution & Increased by \\
\hline \hline 1 & 1.1 & 2.3 & $109 \%$ \\
\hline 2 & 1.4 & 2.5 & $79 \%$ \\
\hline 3 & 1.3 & 2.4 & $85 \%$ \\
\hline 4 & 1.7 & 2.4 & $41 \%$ \\
\hline
\end{tabular}

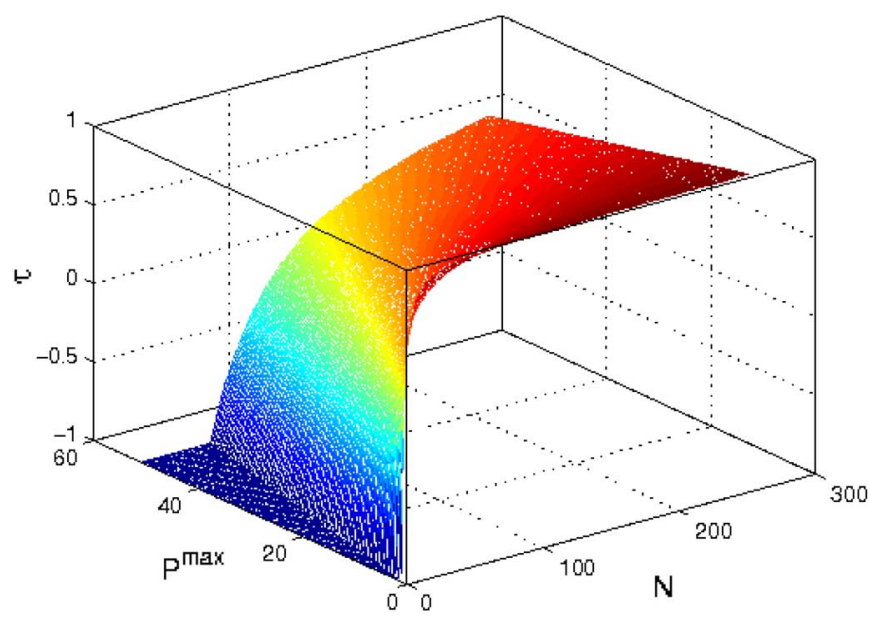

Fig. 4. System classification versus total power limits and number of frequency bins.

$\left.\sum_{k=N-k_{2}+1}^{N} p_{2}^{\max }(k)\right) / p_{2}^{\max }\left(N-k_{2}\right)$ for user 2 . Then the variable $\tau=1-\left(b_{1}+b_{2}\right) / N$ stands for the system characteristic according to Theorem 4 . The system is bandwidth-dominant if $-1 \leq \tau \leq 0$ and it is power-dominant if $0<\tau<1$. It can be seen from Fig. 4 that the system changes gradually from bandwidth- to power-dominant when new frequency bins are added into the system, while it changes gradually from powerto bandwidth-dominant when the total power limits of the users are relaxed.

In our last example, the power-dominant two-user system is considered. The number of frequency bins varies from 4 to 8 (50 runs are used for each case with the number of frequency bins being $4,5,6,7$, and 8 ). The total power limits of the users are set as $P_{i}^{\max }=2\left(i \in \Omega_{M=2}\right)$ for each user, and the power limits on different frequency bins are set to $1+x(k)$ where $x(k)$ is a uniform random variable in the interval [0.2, 0.25]. It guarantees that the system is power-dominant. The channel gains on all frequency bins are randomly generated for both users using Rayleigh distribution with mean 1.

Fig. 5(a) shows the FDM/TS and SJ-FDM/TS based NB solutions $S_{N B}^{o p t}$ and $S_{N B}^{\prime \prime}$, respectively, for all 250 simulation runs. It can be seen in the figure that $S_{N B}^{\prime \prime}$ is identical to $S_{N B}^{o p t}$ for most of the cases. Moreover, although the distance between $S_{N B}^{\prime \prime}$ and $S_{N B}^{o p t}$ for some cases may appear relatively large in the rate region, the differences between the values of the logarithm of their NF are small as shown in Fig. 5(b). Particularly, Fig. 5(b) depicts the logarithm of the NF for $S_{N B}^{o p t}$ and $S_{N B}^{\prime \prime}$ (denoted as NF ${ }^{o p t}$ and $\mathrm{NF}^{\prime \prime}$, respectively) versus the number of frequency bins $N$ when the total power limits $P_{1}^{\max }=P_{2}^{\max }=P^{\max }$ are set to $1.5,2$, or 2.5 . Every point in the figure is averaged over 50 runs. It can be seen from Fig. 5(b) that the gap between $\mathrm{NF}^{o p t}$ and $\mathrm{NF}^{\prime \prime}$ is very small, if not zero. 


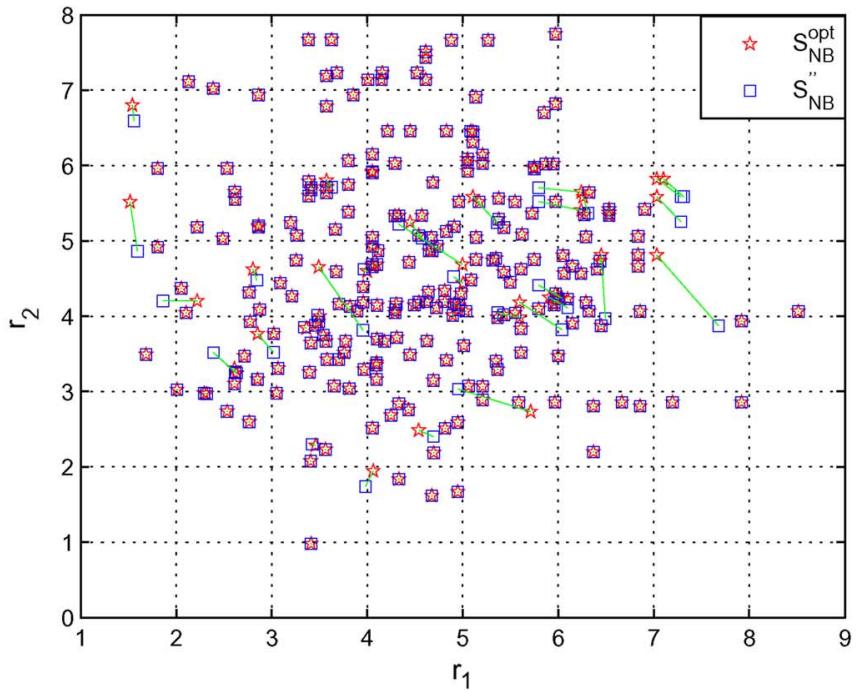

(a)

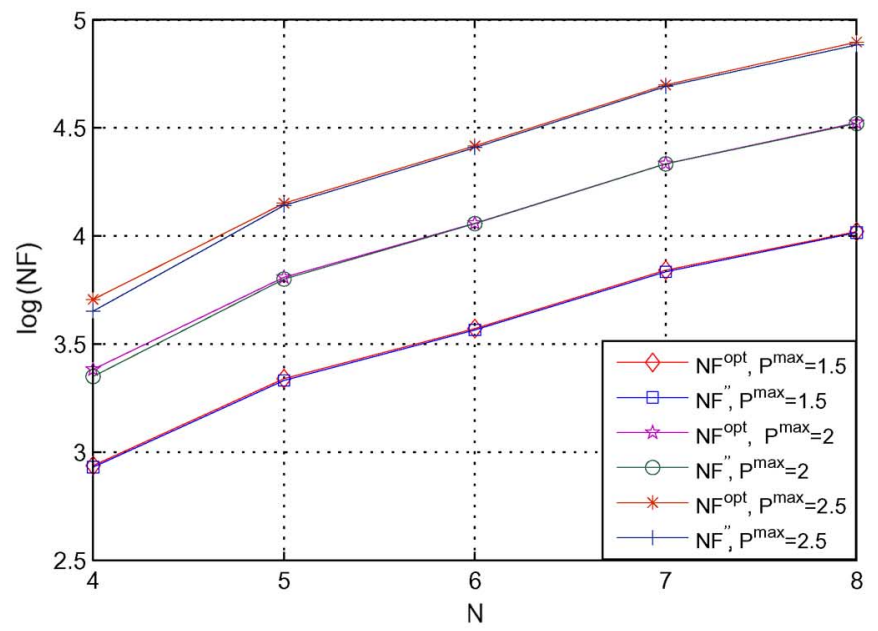

(b)

Fig. 5. Numerical example 3: (a) $S_{N B}^{o p t}$ and $S_{N B}^{\prime \prime}$ in the rate region; (b) the logarithm of the NF for the FDM/TS and SJ-FDM/TS based NB solutions.

\section{CONCLUSIONS}

Cooperative NB based resource allocation strategies have been studied under SMCs and optionally TPCs. First, resource allocation in two-user systems with SMCs has been investigated and then the results have been generalized to $M$-user systems. It has been shown that the joint TDM/FDM is an efficient manner of cooperation for such games. Particularly, it has been shown that the NB based cooperative resource allocation with the joint TDM/FDM based manner of cooperation outperforms the noncooperative resource allocation under mild conditions. A semi-distributed algorithm has been also developed to implement the NB. The two-level structure of the algorithm enables the users to perform most of the computations individually and in parallel using local information.

Second, the NB based cooperative resource allocation game with both SMCs and TPCs has been studied. It has been shown that this game corresponds to a nonconvex optimization problem which requires joint optimization of both the bandwidth (which is the public resource) allocation and the users' individual power allocations. The complexity of finding the optimal solution is unacceptably high in this case. Therefore, it has been proposed to categorize the two-user systems into bandwidth- and power-dominant depending on the bottleneck resource in the system using the concept of Pareto-optimality. Then different manners of cooperation have been suggested for these two classes of systems and corresponding algorithms have been developed. For the bandwidth-dominant systems, bandwidth is the bottleneck resource and, therefore, the proposed algorithm allocates bandwidth as a priority, while a suboptimal power allocation is used to reduce the complexity of the algorithm. Similarly, for the power-dominant systems, power is the bottleneck resource and the proposed algorithm allocates power as a priority, while a suboptimal bandwidth allocation is used to reduce the complexity. This guarantees that the obtained solutions are efficient and can be even identical to the optimal solutions, while the complexity is significantly reduced. Simulation results demonstrate the effectiveness of the proposed cooperative solutions.

\section{APPENDIX A \\ PROOFS OF THEOREMS 1 AND 3 IN SECTION III}

Proof of Theorem 1: Let us prove the per-user condition (20) by first considering the case with $N=1$, i.e., the case when there is only one frequency bin. In this case, the system is a high-interference system if and only if there exists $0<\tilde{\alpha}<1$ which satisfies

$$
\begin{aligned}
\tilde{\alpha} \log \left(1+\frac{p_{1}^{\max }(1)\left|h_{11}(1)\right|^{2}}{\sigma_{1}^{2}(1)}\right) & >r_{1}^{\mathrm{NE}}(1) \\
(1-\tilde{\alpha}) \log \left(1+\frac{p_{2}^{\max }(1)\left|h_{22}(1)\right|^{2}}{\sigma_{2}^{2}(1)}\right) & >r_{2}^{\mathrm{NE}}(1) .
\end{aligned}
$$

These conditions can be rewritten as

$$
\tilde{\alpha} \log \left(1+\frac{p_{1}^{\max }(1)\left|h_{11}(1)\right|^{2}}{\hat{\sigma}_{1}^{2}(1)}\right)>(1-\tilde{\alpha}) r_{1}^{\mathrm{NE}}(1)
$$

$(1-\tilde{\alpha}) \log \left(1+\frac{p_{2}^{\max }(1)\left|h_{22}(1)\right|^{2}}{\hat{\sigma}_{2}^{2}(1)}\right)>\tilde{\alpha} r_{2}^{\mathrm{NE}}(1)$

where

$$
\begin{aligned}
\hat{\sigma}_{i}^{2}(1)= & \sigma_{i}^{2}(1)+\frac{\sigma_{i}^{4}(1)}{p_{j}^{\max }(1)\left|h_{j i}(1)\right|^{2}}+\frac{\sigma_{i}^{2}(1) p_{i}^{\max }(1)\left|h_{i i}(1)\right|^{2}}{p_{j}^{\max }(1)\left|h_{j i}(1)\right|^{2}} \\
= & \sigma_{i}^{2}(1)\left(1+\frac{1+\frac{p_{i}^{\max }(1)\left|h_{i i}(1)\right|^{2}}{\sigma_{i}^{2}(1)}}{\frac{p_{j}^{\max }(1)\left|h_{j i}(1)\right|^{2}}{\sigma_{i}^{2}(1)}}\right) \\
& j \neq i, \quad i \in \Omega_{M=2} .
\end{aligned}
$$

If condition (20) is satisfied for user 1 , that is, $p_{2}^{\max }(1)\left|h_{21}(1)\right|^{2} / \sigma_{1}^{2}(1)>\sqrt{1+p_{1}^{\max }(1)\left|h_{11}(1)\right|^{2} / \sigma_{1}^{2}(1)}$, the

$$
\begin{aligned}
\hat{\sigma}_{1}^{2}(1) & <\sigma_{1}^{2}(1)\left(1+\frac{\left(p_{2}^{\max }(1)\left|h_{21}(1)\right|^{2}\right)^{2} \sigma_{1}^{2}(1)}{p_{2}^{\max }(1)\left|h_{21}(1)\right|^{2} \sigma_{1}^{4}(1)}\right) \\
& =\sigma_{1}^{2}(1)+p_{2}^{\max }(1)\left|h_{21}(1)\right|^{2} .
\end{aligned}
$$


Therefore, the left-hand side of (65) has the following property

$$
\begin{aligned}
& \tilde{\alpha} \log \left(1+\frac{p_{1}^{\max }(1)\left|h_{11}(1)\right|^{2}}{\hat{\sigma}_{1}^{2}(1)}\right) \\
& >\tilde{\alpha} \log \left(1+\frac{p_{1}^{\max }(1)\left|h_{11}(1)\right|^{2}}{\sigma_{1}^{2}(1)+p_{2}^{\max }(1)\left|h_{21}(1)\right|^{2}}\right) \\
& \quad=\tilde{\alpha} r_{1}^{\mathrm{NE}}(1) .
\end{aligned}
$$

It is now easy to verify that there is at least one feasible value for $\tilde{\alpha}$ which satisfies $0<\tilde{\alpha}<1$ and (65), and this value is $\tilde{\alpha}=1 / 2$. In the same way, it can be verified that $\tilde{\alpha}=1 / 2$ also satisfies (66) if (20) is satisfied. Thus, condition (20) is a sufficient condition for the system to be a high-interference system when $N=1$.

The extension to $N>1$ is straightforward. Indeed, from the above proof for $N=1$, it can be seen that if condition (20) is satisfied, then both users can achieve on each frequency bin better utilities than their utilities in the NE by sharing the frequency bins with $\alpha_{1}(k)=\alpha_{2}(k)=0.5\left(\forall k \in \Omega_{N}\right)$, i.e., $\alpha_{i}(k) r_{i}^{\max }(k)=0.5 r_{i}^{\max }(k)>r_{i}^{\mathrm{NE}}(k)\left(\forall i \in \Omega_{M=2}\right)$. Since $r_{i}\left(\boldsymbol{\alpha}_{i}\right)=\sum_{k \in \Omega_{N}} \alpha_{i}(k) r_{i}^{\max }(k)$ and $r_{i}^{\mathrm{NE}}=$ $\sum_{k \in \Omega_{N}} r_{i}^{\mathrm{NE}}(k)\left(\forall i \in \Omega_{M=2}\right)$, it is guaranteed that there is at least one feasible solution for $\alpha_{i}(k)\left(\forall i \in \Omega_{M=2}, \forall k \in \Omega_{N}\right)$, that is, $\alpha_{i}(k)=0.5\left(\forall i \in \Omega_{M=2}, \forall k \in \Omega_{N}\right)$, for which $r_{i}\left(\boldsymbol{\alpha}_{i}\right)>r_{i}^{\mathrm{NE}}\left(\forall i \in \Omega_{M=2}\right)$. Therefore, the domain of the three constraints in (10)-(13) is nonempty and the system is a high-interference system.

Let us now prove the inter-user condition (21) by again first considering the case with $N=1$. The constraints in (10)-(13) have a nonempty domain if and only if there exist $0<\alpha_{1}<1$ and $0<\alpha_{2}<1$ such that

$$
\begin{aligned}
& \alpha_{1} \log \left(1+\frac{p_{1}^{\max }(1)\left|h_{11}(1)\right|^{2}}{\sigma_{1}^{2}(1)}\right) \\
& >\log \left(1+\frac{p_{1}^{\max }(1)\left|h_{11}(1)\right|^{2}}{\sigma_{1}^{2}(1)+p_{2}^{\max }(1)\left|h_{21}(1)\right|^{2}}\right)=r_{1}^{\mathrm{NE}} \\
& \alpha_{2} \log \left(1+\frac{p_{2}^{\max }(1)\left|h_{22}(1)\right|^{2}}{\sigma_{2}^{2}(1)}\right) \\
& >\log \left(1+\frac{p_{2}^{\max }(1)\left|h_{22}(1)\right|^{2}}{\sigma_{2}^{2}(1)+p_{1}^{\max }(1)\left|h_{12}(1)\right|^{2}}\right)=r_{2}^{\mathrm{NE}} \\
& \alpha_{1}+\alpha_{2} \leq 1
\end{aligned}
$$

The conditions (70)-(72) can be written in another form. Specifically, it can be shown that there exist $0<\alpha_{1}<1$ and $0<\alpha_{2}<1$ which satisfy (70)-(72) if and only if there exist $0<\alpha_{1}^{\prime}<1$ and $0<\alpha_{2}^{\prime}<1$ such that

$$
\begin{aligned}
& \alpha_{1}^{\prime} \log \left(1+\frac{p_{1}^{\max }(1)\left|h_{11}(1)\right|^{2}}{\sigma_{1}^{2}(1)}\right) \\
& \quad=\log \left(1+\frac{p_{1}^{\max }(1)\left|h_{11}(1)\right|^{2}}{\sigma_{1}^{2}(1)+p_{2}^{\max }(1)\left|h_{21}(1)\right|^{2}}\right)
\end{aligned}
$$

$$
\begin{aligned}
& \alpha_{2}^{\prime} \log \left(1+\frac{p_{2}^{\max }(1)\left|h_{22}(1)\right|^{2}}{\sigma_{2}^{2}(1)}\right) \\
& =\log \left(1+\frac{p_{2}^{\max }(1)\left|h_{22}(1)\right|^{2}}{\sigma_{2}^{2}(1)+p_{1}^{\max }(1)\left|h_{12}(1)\right|^{2}}\right) \\
& \alpha_{1}^{\prime}+\alpha_{2}^{\prime}<1 .
\end{aligned}
$$

The conditions (73)-(75) are equivalent to the following single condition

$$
\sum_{i \in \Omega_{M=2}} \frac{\log \left(1+\frac{p_{i}^{\max }(1)\left|h_{i i}(1)\right|^{2}}{\sigma_{i}^{2}(1)+p_{j}^{\max }(1)\left|h_{j i}(1)\right|^{2}}\right)}{\log \left(1+\frac{p_{i}^{\max }(1)\left|h_{i i}(1)\right|^{2}}{\sigma_{i}^{2}(1)}\right)}<1, \quad j \neq i .
$$

The left-hand side of (76) is a summation of functions $f(x)=\log (1+x /(1+a)) / \log (1+x)$ with $x=p_{i}^{\max }(1)\left|h_{i i}(1)\right|^{2} / \sigma_{i}^{2}(1)$ and constant $a=p_{j}^{\max }(1)\left|h_{j i}(1)\right|^{2} / \sigma_{i}^{2}(1)>0$. It can be shown that this is an increasing function of $x$ within its domain. Therefore, defining $\gamma_{1}^{0}=$ $\max \left\{p_{1}^{\max }(1)\left|h_{11}(1)\right|^{2} / \sigma_{1}^{2}(1), p_{2}^{\max }(1)\left|h_{22}(1)\right|^{2} / \sigma_{2}^{2}(1)\right\}$, we obtain the following inequality

$$
\begin{aligned}
& \sum_{i \in \Omega_{M=2}} \frac{\log \left(1+\frac{p_{i}^{\max }(1)\left|h_{i i}(1)\right|^{2}}{\sigma_{i}^{2}(1)+p_{j}^{\max }(1)\left|h_{j i}(1)\right|^{2}}\right)}{\log \left(1+\frac{p_{i}^{\max }(1)\left|h_{i i}(1)\right|^{2}}{\sigma_{i}^{2}(1)}\right)} \\
& \leq \sum_{i \in \Omega_{M=2}} \frac{\log \left(1+\frac{\gamma_{1}^{0}}{1+p_{j}^{\max }(1)\left|h_{j i}(1)\right|^{2} / \sigma_{i}^{2}(1)}\right)}{\log \left(1+\gamma_{1}^{0}\right)}, \quad j \neq i
\end{aligned}
$$

which is satisfied as equality if and only if $p_{1}^{\max }(1)\left|h_{11}(1)\right|^{2} / \sigma_{1}^{2}(1)=p_{2}^{\max }(1)\left|h_{22}(1)\right|^{2} / \sigma_{2}^{2}(1)$.

Therefore, if

$$
\begin{aligned}
& \sum_{i \in \Omega_{M=2}} \frac{\log \left(1+\frac{\gamma_{1}^{0}}{1+\frac{p_{j}^{\max }(1)\left|h_{j i}(1)\right|^{2}}{\sigma_{i}^{2}(1)}}\right)}{\log \left(1+\gamma_{1}^{0}\right)} \\
& =\frac{\sum_{i \in \Omega_{M=2}} \log \left(1+\frac{\gamma_{1}^{0}}{1+\frac{p_{j}^{\max }(1)\left|h_{j i}(1)\right|^{2}}{\sigma_{i}^{2}(1)}}\right)}{\log \left(1+\gamma_{1}^{0}\right)} \\
& <1, \quad j \neq i
\end{aligned}
$$

or, equivalently, if

$$
\prod_{i \in \Omega_{M=2}}\left(1+\frac{\gamma_{1}^{0}}{1+\frac{p_{j}^{\max }(1)\left|h_{j i}(1)\right|^{2}}{\sigma_{i}^{2}(1)}}\right)<1+\gamma_{1}^{0}, \quad j \neq i
$$

then (76) is guaranteed to be satisfied and the system is a highinterference system.

The extension to $N>1$ is again straightforward. Indeed, the $\mathrm{NE}$ rate of the game for user $i$ is a summation of its NE rates on each frequency bin, i.e., $r_{i}^{\mathrm{NE}}=\sum_{k \in \Omega_{N}} r_{i}^{\mathrm{NE}}(k)$. The overall rate given by the joint TDM/FDM based NB solution for user $i$ is also a summation of its rates on each frequency bin, i.e., 
$r_{i}\left(\boldsymbol{\alpha}_{i}\right)=\sum_{k \in \Omega_{N}} \alpha_{i}(k) r_{i}^{\max }(k)$. Therefore, if the domain of the following per-bin constraints

$$
\begin{aligned}
\alpha_{i}(k) r_{i}^{\max }(k) & >r_{i}^{\mathrm{NE}}(k), \quad \forall i \in \Omega_{M=2} \\
0 & <\alpha_{i}(k)<1, \quad \forall i \in \Omega_{M=2} \\
\alpha_{1}(k)+\alpha_{2}(k) & \leq 1
\end{aligned}
$$

is nonempty for each frequency bin $k\left(\forall k \in \Omega_{N}\right)$, then the domain of the constraints in the problem (10)-(13) is also nonempty. This completes the proof.

Proof of Theorem 3: Since the constraints of the problem (23)-(26) are all linear, the Slater's condition reduces to two parts with the first part requiring that the feasible domain of $f=$ $\sum_{i \in \Omega_{M}} \log \left(r_{i}\left(\boldsymbol{\alpha}_{i}\right)-r_{i}^{\mathrm{NE}}\right)$ be open and the second part requiring that the feasible domain of the whole problem be nonempty.

It is straightforward to verify that the first part is satisfied. The second part is equivalent to the requirement of the existence of the NB solution. This completes the proof.

\section{APPENDIX B}

\section{PROOFS OF THEOREMS 4 TO 7 IN SECTION IV}

Proof of Theorem 4: First note that in game $\mathcal{G} 1$ any resource allocation scheme satisfying (50)-(52) results in a Pareto-optimal point in the utility space, and vice versa. Thus, the statement of the theorem is equivalent to the statement that (53) is the sufficient and necessary condition which guarantees that at least one set of $\{\hat{k}, \beta\}$ satisfies the conditions (50)-(52). To prove the sufficiency, let (53) be satisfied, $\hat{k}=\tilde{k}$, and $\beta=\tilde{\alpha}$ in (50)-(52). Then the resulted total powers used by the users are $P_{1}^{\prime}=\sum_{k=1}^{\tilde{k}-1} p_{1}^{\max }(k)+\tilde{\alpha} p_{1}^{\max }(\tilde{k})$ for user 1 and $P_{2}^{\prime}=$ $\sum_{k=\tilde{k}+1}^{N} p_{2}^{\max }(k)+(1-\tilde{\alpha}) p_{2}^{\max }(\tilde{k})$ for user 2. Using (53), it is easy to verify that $P_{1}^{\max } \geq P_{1}^{\prime}$ and $P_{2}^{\max } \geq P_{2}^{\prime}$. Therefore, the sufficiency is proved. The necessity can be proved similarly using contradiction.

Proof of Theorem 5: The first part of this theorem follows from the independence on irrelevant alternatives property of the NB [24]. This property states that bargaining in a convex subset which contains the NB solution of the original set results in the same NB solution. Thus, if $S_{N B}^{o p t} \in \mathcal{P}^{\prime}$, then $S_{N B}^{\prime}=S_{N B}^{o p t}$. Therefore, it is clear that if $S_{N B}^{\prime} \neq S_{N B}^{o p t}$, then $S_{N B}^{o p t} \notin \mathcal{P}^{\prime}$. Since $\mathcal{P}^{\prime}$ is the achievable subset of $\mathcal{P}_{1}$ in game $\mathcal{G} 2$, it is impossible that $S_{N B}^{o p t} \in \mathcal{P}_{1}$ and $S_{N B}^{o p t} \notin \mathcal{P}^{\prime}$ simultaneously. Thus, if $S_{N B}^{o p t} \notin$ $\mathcal{P}^{\prime}$, then $S_{N B}^{o p t} \notin \mathcal{P}_{1}$ as well. This completes the proof.

Proof of Theorem 6: First, note that the line segment $\left[r_{1}^{d}, r_{1}^{u}\right]$ is the projection of the boundary $\mathcal{P}^{\prime}$ onto the abscissa, i.e., onto $r_{1}$ axis. To prove this, we only need to verify that the minimum and maximum rates that user 1 can obtain on $\mathcal{P}^{\prime}$ are $r_{1}^{d}$ and $r_{1}^{u}$, respectively. From (55) and (56), it can be seen that the minimum rate that user 1 can obtain on $\mathcal{P}^{\prime}$ is indeed $r_{1}^{d}$ when user 1 occupies frequency bins 1 to $\breve{k}-2$ exclusively and frequency bin $\breve{k}-1$ for a $\left(1-\left(P_{2}^{\max }-\sum_{k=\breve{k}}^{N} p_{2}^{\max }(k)\right) / p_{2}^{\max }(\breve{k}-1)\right)$ portion of time. Similarly, from (57) and (58), it can be seen that the maximum rate that user 1 can obtain on $\mathcal{P}^{\prime}$ is indeed $r_{1}^{u}$ when user 1 occupies frequency bins 1 to $\bar{k}$ exclusively and frequency bin $\bar{k}+1$ for a $\left(P_{1}^{\max }-\sum_{k=1}^{\bar{k}} p_{1}^{\max }(k)\right) / p_{1}^{\max }(\bar{k}+1)$ portion of time. Note that using (53) in Theorem 4, it can be shown that $r_{1}^{d} \leq r_{1}^{u}$ for bandwidth-dominant systems.

Second, according to Theorem 5, a sufficient condition for $S_{N B}^{\prime}=S_{N B}^{o p t}$ is that the optimal solution of game $\mathcal{G} 1$ is in $\mathcal{P}^{\prime}$. Recall that the Pareto-boundary $\mathcal{P}_{1}$ of $\mathcal{U}_{1}$ is a piece-wise linear boundary with $N$ segments $l_{1}, \ldots, l_{N}$ and $N-1$ junctions $J_{1}, \ldots, J_{N-1}$. The slop of segment $k$ is $-r_{2}^{\max }(k) / r_{1}^{\max }(k)\left(k \in \Omega_{N}\right)$, while the rates associated with junction $J_{t}$ are $r_{1}^{J_{t}}$ and $r_{2}^{J_{t}}$ for users 1 and 2, respectively. Denoting the optimal solution of game $\mathcal{G} 1$ associated with rates $r_{1}^{\star}$ and $r_{2}^{\star}$ as $S_{N B}^{\star} \in \mathcal{P}_{1}$, it can be shown that the point $\left(r_{1}^{\star}, r_{2}^{\star}\right)$ can be either in the interior of one of the line segments $l_{k}\left(k \in \Omega_{N}\right)$ or at one of the junctions $J_{t}\left(t \in \Omega_{N}-\{N\}\right)$. If $\left(r_{1}^{\star}, r_{2}^{\star}\right)$ is in the interior of one of the line segments $l_{k}\left(k \in \Omega_{N}\right)$, there is a Nash curve, that is, $r_{1} \cdot r_{2}=c$, which is tangent to the convex rate region, for a unique constant $c$. More specifically, it is tangent to one of the line segments $l_{k}\left(k \in \Omega_{N}\right)$ at the point $\left(r_{1}^{*}, r_{2}^{*}\right)$. Note that it is possible that the Nash curve is tangent to the convex rate region at one of the junctions as well, which provides rates $r_{1}^{\star}$ and $r_{2}^{\star}$ for users 1 and 2, respectively. Moreover, in the case when there is no tangent point, there exists a Nash curve, that is, $r_{1} \cdot r_{2}=c^{\prime}$, that intersects with the rate region only at $\left(r_{1}^{\star}, r_{2}^{\star}\right)$, which is one of the junctions $J_{t}\left(t \in \Omega_{N}-\{N\}\right)$, for a unique constant $c^{\prime}$.

Let the point $\left(r_{1}^{0}, r_{2}^{0}\right)$ on the line segment $l_{k}$ be the tangent point, then the corresponding Nash curve is $r_{1} \cdot r_{2}=r_{1}^{0} \cdot r_{2}^{0}$ with the slope at $\left(r_{1}^{0}, r_{2}^{0}\right)$ being $-r_{2}^{0} / r_{1}^{0}$. At the same time, the slope of the line segment $l_{k}$ is $-r_{2}^{\max }(k) / r_{1}^{\max }(k)$. Thus, if $\left(r_{1}^{0}, r_{2}^{0}\right)$ is indeed a tangent point, then $-r_{2}^{\max }(k) / r_{1}^{\max }(k)=-r_{2}^{0} / r_{1}^{0}$, and the first line of (54) is proved. Another case is when $S_{N B}^{\star}$ is a unique intersection point at one of the junctions $J_{t}(t \in$ $\left.\Omega_{N}-\{N\}\right)$. In this case, the optimal solution maximizes $r_{1}^{J_{t}} \cdot r_{2}^{J_{t}}$ within all $N-1$ junctions, and the second line of (54) straightforwardly follows.

It is now easy to see that $S_{N B}^{\star} \in \mathcal{P}^{\prime}$ if and only if $r_{1}^{\star} \in$ $\left[r_{1}^{d}, r_{1}^{u}\right]$. This completes the proof.

Proof of Theorem 7: Let $r_{1}^{\text {opt } 1}>r_{1}^{\text {opt } 2}$. Then $r_{2}^{o p t 1}<r_{2}^{o p t 2}$ due to the Pareto-optimality. Let also $\mathbf{r}^{1}=\left(r_{1}^{1}, r_{2}^{1}\right)$ and $\mathbf{r}^{2}=$ $\left(r_{1}^{2}, r_{2}^{2}\right)$ be two points generated in Steps 2 and 3 of the algorithm summarized in Table II such that $r_{1}^{1} \geq r_{1}^{\text {opt } 1}$ and $r_{2}^{2} \geq r_{2}^{\text {opt } 2}$. Denote the sets of frequency bins allocated to the users 1 and 2 in the points $\mathbf{r}^{1}$ and $\mathbf{r}^{2}$ as $\left(\mathcal{B}_{1}^{1}, \mathcal{B}_{2}^{1}\right)$ and $\left(\mathcal{B}_{1}^{2}, \mathcal{B}_{2}^{2}\right)$, respectively, where the superscripts stand for the corresponding indexes of the points and the subscripts denote the corresponding indexes of the users. Recalling that $r_{i}^{\prime}=0\left(\forall i \in \Omega_{M}\right)$, i.e., the disagreement point is the origin, the difference between the logarithm of the NF for $\mathbf{r}^{o p t}$ and the logarithm of the NF for $\mathbf{r}^{1}$ can be obtained as

$$
\begin{aligned}
d_{1}= & \log \left(\lambda r_{1}^{\text {opt } 1}+(1-\lambda) r_{1}^{\text {opt } 2}\right) \\
& +\log \left(\lambda r_{2}^{\text {opt } 1}+(1-\lambda) r_{2}^{\text {opt } 2}\right)-\log \left(r_{1}^{1}\right)-\log \left(r_{2}^{1}\right) \\
= & \log \left(\lambda \frac{r_{1}^{\text {opt } 1}}{r_{1}^{1}}+(1-\lambda) \frac{r_{1}^{\text {opt } 2}}{r_{1}^{1}}\right) \\
& +\log \left(\lambda \frac{r_{2}^{\text {opt } 1}}{r_{2}^{1}}+(1-\lambda) \frac{r_{2}^{\text {opt }} 2}{r_{2}^{1}}\right)
\end{aligned}
$$




$$
\begin{aligned}
& <\log \left(\lambda \frac{r_{1}^{o p t 1}}{r_{1}^{1}}+(1-\lambda) \frac{r_{1}^{o p t 1}}{r_{1}^{1}}\right) \\
& +\log \left(\lambda \frac{r_{2}^{o p t 2}}{r_{2}^{1}}+(1-\lambda) \frac{r_{2}^{o p t 2}}{r_{2}^{1}}\right) \\
& \leq \log \left(\lambda \frac{r_{2}^{o p t 2}}{r_{2}^{1}}+(1-\lambda) \frac{r_{2}^{o p t 2}}{r_{2}^{1}}\right) \\
& =\log \left(\frac{r_{2}^{o p t 2}}{r_{2}^{1}}\right)=\log \left(\frac{\mathcal{W} \mathcal{F}^{2}\left(\mathcal{B}_{2}^{o p t 2}\right)}{\mathcal{W} \mathcal{F}^{2}\left(\mathcal{B}_{2}^{1}\right)}\right)
\end{aligned}
$$

where the inequalities hold because $\log \left(\lambda r_{1}^{o p t 1}+(1-\lambda) r_{1}^{o p t 2}\right)+$ $\log \left(\lambda r_{2}^{o p t 1}+(1-\lambda) r_{2}^{o p t 2}\right)-\log \left(r_{1}^{1}\right)-\log \left(r_{2}^{1}\right)>0, r_{1}^{o p t 1}>$ $r_{1}^{o p t 2}, r_{2}^{o p t 1}<r_{2}^{o p t 2}$, and $r_{1}^{1} \geq r_{1}^{o p t 1}$, and the last equality is obtained by substituting the notations $r_{2}^{\text {opt } 2}=\mathcal{W} \mathcal{F}^{2}\left(\mathcal{B}_{2}^{\text {opt } 2}\right)$ and $r_{2}^{1}=\mathcal{W}^{2} \mathcal{F}^{2}\left(\mathcal{B}_{2}^{1}\right)$. Furthermore, using the fact that $\mathcal{W} \mathcal{F}^{2}\left(\mathcal{B}_{2}^{1}\right) \geq$ $\mathcal{W} \mathcal{F}^{2}\left(\mathcal{B}-\tilde{\mathcal{B}}_{1}\right),(83)$ can be simplified as

$$
d_{1}<\log \left(\frac{\mathcal{W} \mathcal{F}^{2}\left(\mathcal{B}_{2}^{o p t 2}\right)}{\mathcal{W} \mathcal{F}^{2}\left(\mathcal{B}-\tilde{\mathcal{B}}_{1}\right)}\right) .
$$

It can be derived in a similar way that the difference $d_{2}$ between the logarithm of the NF for $\mathbf{r}^{o p t}$ and the logarithm of the NF for $\mathbf{r}^{2}$ obeys the following inequality

$$
d_{2}<\log \left(\frac{\mathcal{W} \mathcal{F}^{1}\left(\mathcal{B}_{1}^{\text {opt } 1}\right)}{\mathcal{W} \mathcal{F}^{1}\left(\mathcal{B}-\tilde{\mathcal{B}}_{2}\right)}\right)
$$

Finally, note that neither $\mathbf{r}^{1}$ nor $\mathbf{r}^{2}$ has been assumed to be the rates corresponding to the SJ-FDM/TS based NB solution. Indeed, $\mathbf{r}^{1}$ and $\mathbf{r}^{2}$ are just two of $2 L$ points generated in Steps 2 and 3 of the algorithm summarized in Table II, respectively. Thus, the rates corresponding to the actual solution returned by the algorithm are expected to be superior to the rates corresponding to the points $\mathbf{r}^{1}$ and $\mathbf{r}^{2}$, or equal to the rates in the optimal solution $\mathbf{r}^{o p t}=\left(\lambda r_{1}^{o p t 1}+(1-\lambda) r_{1}^{o p t 2}, \lambda r_{2}^{o p t 1}+(1-\lambda) r_{2}^{o p t 2}\right)$. As an example, when $\left|\mathcal{B}_{c}\right|=0$, the solution obtained by the algorithm in Table II is identical to the optimal solution $\mathbf{r}^{o p t}$. Therefore, $d \leq \min \left(d_{1}, d_{2}\right)$ and $d$ can be equal to zero. This completes the proof.

\section{ACKNOWLEDGMENT}

The authors would like to thank the Associate Editor Dr. W. Hachem of Telecom ParisTech for his useful comment on a quantified characterization of interference level which motivated part of the contribution in Section III.

\section{REFERENCES}

[1] F. Meshkati, V. Poor, and S. Schwartz, "Energy-efficient resource allocation in wireless networks: An overview of game-theoretic approaches," IEEE Signal Process. Mag., vol. 24, no. 3, pp. 58-68, May 2007.

[2] H. Yaiche, R. R. Mazumdar, and C. Rosenberg, "A game theoretic framework for bandwidth allocation and pricing in broadband networks," IEEE/ACM Trans. Netw., vol. 8, no. 5, pp. 667-678, Oct. 2000.

[3] Z. Han, Z. Ji, and K. J. R. Liu, "Fair multiuser channel allocation for OFDMA networks using Nash bargaining solutions and coalitions," IEEE Trans. Commun., vol. 53, no. 8, pp. 1366-1376, Aug. 2005.
[4] W. Yu, G. Ginis, and J. Cioffi, "Distributed multiuser power control for digital subscriber lines," IEEE J. Sel. Areas Commun., vol. 20, no. 5, pp. 1105-1115, Jun. 2002

[5] Z. Q. Luo and J. S. Pang, "Analysis of iterative waterfilling algorithm for multi-user power control in digital subscriber lines," EURASIP J. Appl. Signal Process. 2009 [Online]. Available: http://downloads.hindawi.com/journals/asp/2006/024012.pdf

[6] E. V. Belmega, S. Lasaulce, and M. Debbah, "Power allocation games for MIMO multiple access channels with coordination," IEEE Trans. Wireless Commun., vol. 8, no. 6, pp. 3182-3192, Jun. 2009.

[7] M. Bennis, M. L. Treust, S. Lasaulce, M. Debbah, and J. Lilleberg, "Spectrum sharing games on the interference channel," in Proc. Int. GameNets Conf., Istanbul, Turkey, May 2009.

[8] D. Niyato and E. Hossain, "Competitive spectrum sharing in cognitive radio networks: A dynamic game approach," IEEE Trans. Wireless Commun., vol. 7, no. 7, pp. 2651-2660, Jul. 2008.

[9] E. G. Larsson and E. A. Jorswieck, "Competition versus cooperation on the MISO interference channel," IEEE J. Sel. Areas Commun., vol. 26, no. 7, pp. 1059-1069, Sep. 2008.

[10] A. Leshem and E. Zehavi, "Bargaining over the interference channel," in Proc. IEEE Int. Symp. Inf. Theory, Seattle, WA, Jul. 2006, pp. 2225-2229.

[11] A. Leshem and E. Zehavi, "Cooperative game theory and the Gaussian interference channel,” IEEE J. Sel. Areas Commun., vol. 26, no. 7, pp. 1078-1088, Sep. 2008.

[12] E. Zehavi and A. Leshem, "Bargaining over the interference channel with total power constraints," in Proc. Int. GameNets Conf., Istanbul, Turkey, May 2009.

[13] H. Boche and M. Schubert, "Nash bargaining and proportional fairness for wireless systems," IEEE/ACM Trans. Netw., vol. 17, no. 5, pp. 1453-1466, Oct. 2009.

[14] E. A. Jorswieck, E. G. Larsson, and D. Danev, "Complete characterization of the Pareto boundary for the MISO interference channel," IEEE Trans. Signal Process., vol. 56, no. 10, pp. 5292-5296, Oct. 2008.

[15] J. Gao, S. A. Vorobyov, and H. Jiang, "Game theoretic solutions for precoding strategies over the interference channel," in Proc. IEEE Global Telecommun. Conf., New Orleans, LA, Nov.-Dec. 2008.

[16] J. Nash, "Two-person cooperative games," Econometrica, vol. 21, no. 1, pp. 128-140, Jan. 1953.

[17] J. Gao, S. A. Vorobyov, and H. Jiang, "Game theory for precoding in a multi-user system: Bargaining for overall benefits," in Proc. IEEE Int. Conf. Acoustics, Speech, Signal Processing, Taipei, Taiwan, Apr. 2009, pp. 2361-2364.

[18] J. Gao, S. A. Vorobyov, and H. Jiang, "Pareto-optimal solutions of Nash bargaining games for resource allocation in wireless systems," in Proc. IEEE Int. Conf. Acoustics, Speech, Signal Processing, Dallas, TX, Mar. 2010, pp. 3146-3149.

[19] P. Vandewalle, J. Kovacevic, and M. Vetterli, "Reproducible research in signal processing," IEEE Signal Process. Mag., vol. 26, no. 3, pp. 37-47, May 2009.

[20] Z. Chen, S. A. Vorobyov, C.-X. Wang, and J. Thompson, "Nash bargaining over MIMO interference systems," in Proc. IEEE Int. Conf. Commun., Dresden, Germany, Jun. 2009.

[21] D. P. Bertsekas, Nonlinear Programming, 2nd ed. Belmont, MA: Athena Scientific, 1999.

[22] S. Boyd and L. Vandenberghe, Convex Optimization. Cambridge, U.K.: Cambridge Univ. Press, 2004

[23] S. C. Liew and Y. J. Zhang, "Proportional fairness in multi-channel multi-rate wireless networks-Part I: The case of deterministic channels with application to AP association problem in large-scale WLAN," IEEE Trans. Wireless Commun., vol. 7, no. 9, pp. 3446-3456, Sep. 2008.

[24] D. Fudenberg and J. Tirole, Game Theory. Cambridge, MA: MIT Press, 1991.

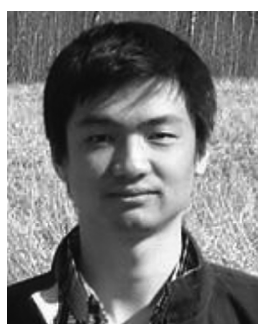

Jie Gao (S'09) received the B.Eng. degree in electronics and information engineering from Huazhong University of Science and Technology, Wuhan, China, in 2007 and the M.Sc. degree in electrical engineering from the University of Alberta, Edmonton, $\mathrm{AB}$, Canada, in 2009. He is currently working towards the Ph.D. degree at the University of Alberta.

His research interests include applications of optimization and game theoretic methods in signal processing and communications, and cross-layer design for wireless multimedia communications. 


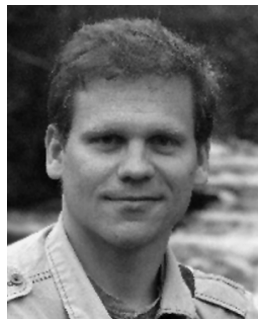

Sergiy A. Vorobyov (M'02-SM'05) received the M.S. and Ph.D. degrees in systems and control from Kharkiv National University of Radioelectronics, Ukraine, in 1994 and 1997, respectively.

Since 2006, he has been with the Department of Electrical and Computer Engineering, University of Alberta, Edmonton, AB, Canada, where he became an Associate Professor in 2010. Since his graduation, he also occupied various research and faculty positions in the Kharkiv National University of Radioelectronics, Ukraine; the Institute of Physical and Chemical Research (RIKEN), Japan; the McMaster University, Canada; Duisburg-Essen and Darmstadt Universities, Germany; and the Joint Research Institute, Heriot-Watt and Edinburgh Universities, U.K. His research interests include statistical and array signal processing, applications of linear algebra, optimization, and game theoretic methods in signal processing and communications, estimation and detection theory, sampling theory, and cooperative and cognitive systems.

Dr. Vorobyov is a recipient of the 2004 IEEE Signal Processing Society Best Paper Award, the 2007 Alberta Ingenuity New Faculty Award, and other research awards. He was an Associate Editor for the IEEE SIGNAL Processing LeTTERS (2007-2009). He has been an Associate Editor for the IEEE TRANSACTIONS ON Signal Processing since 2006. He is a member of the Sensor Array and Multi-Channel Signal Processing Technical Committee of IEEE Signal Processing Society.

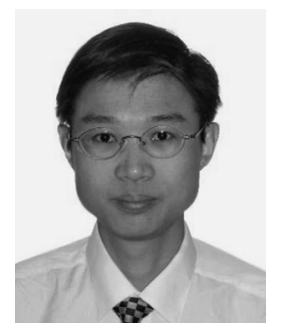

Hai Jiang (M'07) received the B.Sc. and M.Sc. degrees in electronics engineering from Peking University, Beijing, China, in 1995 and 1998, respectively, and the Ph.D. degree (with an Outstanding Achievement in Graduate Studies Award) in electrical engineering from the University of Waterloo, Waterloo, ON, Canada, in 2006.

Since July 2007, he has been an Assistant Professor with the Department of Electrical and Computer Engineering, University of Alberta, Edmonton, AB, Canada. His research interests include radio resource management, cognitive radio networking, and cross-layer design for wireless multimedia communications.

Dr. Jiang is an Associate Editor for the IEEE TRANSACTIONS ON VEHICULAR TECHNOLOGY. He served as a Co-Chair for the General Symposium at the International Wireless Communications and Mobile Computing Conference in 2007, the Communications and Networking Symposium at the Canadian Conference on Electrical and Computer Engineering in 2009, and the Wireless and Mobile Networking Symposium at the IEEE International Conference on Communications in 2010. He received an Alberta Ingenuity New Faculty Award in 2008 and a Best Paper Award from the IEEE Global Communications Conference (GLOBECOM) in 2008. 